\title{
Mut zur Reform: „Hartz IV“ als Testfall für eine zukunftsfähige Arbeits- und Sozialordnung
}

\author{
von Joachim Jens Hesse und Alexander Götz
}

\begin{abstract}
Die Zusammenführung von Arbeitslosen- und Sozialhilfe, einem breiteren Publikum unter der Bezeichnung „Hartz IV“ bekannt, stellt nicht nur eine für die Bundesrepublik grundlegende sozialpolitische Neuorientierung, sondern mit Blick auf die Aufgabenträgerschaft auch eine umfassende Verwaltungsreform dar. Ein wesentliches Element dieser Reform, die Bildung sog. „Arbeitsgemeinschaften“ aus Gliederungen der Bundesagentur für Arbeit und der Kommunen, wurde 2007 durch das Bundesverfassungsgericht als unzulässige Mischverwaltung verworfen. Die hier vorgestellten Ergebnisse einer mehrjährigen flächendeckenden Evaluation des Reformansatzes und seiner Wirkungen verdeutlichen die systematischen Vor-wie Nachteile der einzelnen Trägermodelle. Trotz deutlicher Vorzüge des sog. „Optionsmodells" votieren die Autoren aus staatspolitischen, fiskalischen und funktionalen Gründen für einen allerdings optimierten und deutlich dezentral geprägten Systemwettbewerb.
\end{abstract}

By combining long-term unemployed benefits and social welfare (known as "Hartz IV"), Germany adopted a far-reaching reform of its labour market policies. The approach implied not only a substantial re-orientation of social policy, but also an ambitious administrative reform. A key element of the latter, amalgamating units of the Federal Employment Agency and local authorities, has been rejected by the Federal Constitutional Court in 2007. The results of a comprehensive long-term evaluation of the reform approach, as presented here, demonstrate the pros and cons of different organisational options to cope with this specific target group. Despite their preference for a more decentralised organisational model, the authors refer to the continuing importance of an optimised systemic competition.

\section{Einführung und Ergebniszusammenfassung}

\section{Das SGB II als tiefgreifende Veränderung des deutschen Sozialstaats}

Das Vierte Gesetz für moderne Dienstleistungen am Arbeitsmarkt und die Einführung des Zweiten Sozialgesetzbuchs (SGB II) markierte nicht nur eine tiefgreifende Veränderung des deutschen Sozialstaates, sondern beinhaltete auch 
eine für die Geschichte der Bundesrepublik in Teilen beispiellose Verwaltungsreform. Mit der flächendeckenden Bildung von Aufgabenträgern ${ }^{1}$ der Grundsicherung für Arbeitsuchende sahen sich der Bund, die ihm nachgeordnete Bundesagentur für Arbeit, in Teilen auch die Länder, vor allem aber die Kommunen vor grundlegende organisatorische Herausforderungen gestellt. Die Durchdringung der neuen Rechtsmaterie, die in ihrer gesetzlichen Ausgestaltung von Anfang an strittige und Ende 2007 durch das Bundesverfassungsgericht verworfene Zusammenarbeit ${ }^{2}$ mit einer zentralen Bundeseinrichtung, die Einigung über örtliche Lösungen, der Aufbau arbeitsfähiger Kapazitäten sowie die Überführung von Anspruchsberechtigten und des dem zugrunde liegenden Datenbesatzes mussten parallel in kürzester Zeit vollzogen werden. Der aus Sicht der Leistungsempfänger weitgehend reibungslose Übergang in ein neues Hilfe- und Betreuungssystem zum 1. Januar 2005 ist insofern auch ein Ausweis der Reformund Anpassungsfähigkeit der beteiligten Institutionen.

Zugleicht verweist die organisationspolitische Dimension der Reform auf wesentliche Erfolgs- und Kontextbedingungen einer effektiven und effizienten Arbeitsmarktpolitik. Dies betrifft zunächst die administrativen Kapazitäten und Routinen vor Ort, die mindestens ebenso stark wie rechtlich begründete Instrumente und Anreizstrukturen die Wirkung des SGB II konditionieren dürften. So zeigen nicht zuletzt auch ausländische Erfahrungen, dass eine verbesserte Vermittlung und Integration von Langzeitarbeitslosen offenbar wesentlich vom personellen Einsatz und dem $\mathrm{Ma} ß$ an individueller Betreuung durch die zuständigen Einrichtungen abhängen. Damit verbindet sich keine pauschale Kritik an ggf. zu ausdifferenzierten und einem in der Vergangenheit häufig eher mechani-

1 Gem. § 6 Abs. 1 SGB II sind die Bundesagentur für Arbeit sowie die Kreise und kreisfreien Städte Aufgabenträger der Grundsicherung für Arbeitsuchende. Im Fall der Bildung von ARGEn nach $\S 44 \mathrm{~b}$ wurden diese (ungeachtet des zwischenzeitlich zur Ihrer Verfassungswidrigkeit ergangenen Urteils) zunächst nur zur einheitlichen Aufgabenwahrnehmung errichtet, womit sie nicht den Charakter von Aufgabenträgern im juristischen Sinne erhielten. Aus semantischen Gründen sowie mit Blick auf die sich in der Realität dem Profil einer eigenständigen Behörde annähernden Situation der ARGEn spricht der Gutachter aber auch in ihrem Fall von Aufgabenträgern, womit gleichwohl nicht die gesetzlichen Vorgaben und damit verbundene Rechtsverhältnisse in Abrede gestellt oder etwaige Änderungen präjudiziert werden sollen.

2 BVerfG, Urteil vom 20.12. 2007, 2 BvR 2433/04; vgl. dazu auch die im Rahmen der mündlichen Verhandlung auf der Basis der hier zugrundeliegenden Evaluation vorgetragenen Einschätzungen: Hesse, J.J.: Aufgabenwahrnehmung in den ARGEn nach § 44b SGB II. Umsetzung gesetzlicher Vorgaben, Entscheidungsstrukturen und mögliche Defizite. Mündliche Verhandlung zu den kommunalen Verfassungsbeschwerden 2 BvR 2433/04 und 2 BvR 2434/04, Bundesverfassungsgericht, Karlsruhe, 24.05. 2007; Nachdruck in Hesse, J.J/Götz, A.: Für eine zukunftsfähige Arbeits- und Sozialverwaltung, Baden-Baden, 2007, 213-224. 
schen Aktivierungsverständnis. Vielmehr sollte deutlich werden, dass allgemein zugängliche Dienstleistungen als Kernkompetenz der öffentlichen Hand personell untersetzt und kundenbezogen ausgerichtet werden müssen (um i.S. des SGB II zu wirken), hinter einer mittel- und langfristig erfolgreichen Umsetzung von „Hartz IV“ also immer auch eine entsprechende Organisationsleistung der verantwortlichen Träger und Grundsicherungsstellen steht.

Ferner ist zu berücksichtigen, dass die Umsetzung des SGB II nicht losgelöst von den gegebenen institutionellen Rahmenbedingungen zu sehen ist. Bedeutung erlangen hier vor allem inhaltliche und funktionale Wechselwirkungen zwischen den Aufgaben und den spezifischen Kompetenzen der beteiligten Einrichtungen. Dies betrifft deren besondere Eignung ebenso wie die gegebene Leistungsfähigkeit in anderen Zuständigkeitsbereichen. In Anbetracht einer zunehmenden Entgrenzung als Konsequenz aus „Globalisierung“ und „Europäisierung“ sowie der anhaltenden Krise der öffentlichen Haushalte verbinden sich mit der Zusammenlegung von Arbeitslosen- und Sozialhilfe deshalb nicht nur fachliche Chancen, sondern auch beträchtliche Belastungen, ggf. sogar Risiken. So sehen sich gerade die kommunalen Gebietskörperschaften einer erkennbar an Dynamik gewinnenden Diskussion von Funktional- und Strukturreformen auf Länderebene ausgesetzt, deren Folgen sie ebenfalls zu bewältigen haben. ${ }^{3}$ Blickt man auf die beiden

3 Vgl. hierzu unter den neueren Arbeiten der Autoren: Hesse, J. J.: Regierungs- und Verwaltungsreform in Nordrhein-Westfalen, Düsseldorf/Berlin, 1999; ders.: Regierungs- und Verwaltungsreform in Brandenburg, Potsdam/Berlin, 1999; ders.: Regierungs- und Verwaltungsreform in Schleswig-Holstein, Kiel/ Berlin, 2000; ders.: Regierungs- und Verwaltungsreform in Mecklenburg-Vorpommern, Schwerin/Berlin, 2000; ders.: Regierungs- und Verwaltungsreform in Rheinland-Pfalz, Mainz/Berlin, 2000; ders.: Regierungs- und Verwaltungsreform in Sachsen-Anhalt, Magdeburg/Berlin, 2001; ders.: Regierungsund Verwaltungsreform in Baden-Württemberg, Stuttgart/Berlin, 2002; ders.: Regierungs- und Verwaltungsreform in Bayern, München/Berlin, 2002; ders.: Staatsreform in Deutschland - das Beispiel Hessen, Wiesbaden/Berlin, 2002 (aktualisierte Fassung der gemeinsam mit T. Ellwein 1997 vorgestellten Untersuchung); ders.: Regierungs- und Verwaltungsreform in Nordrhein-Westfalen. Eine Zwischenbilanz und Bewertung von Regierungs- wie Oppositionsvorschlägen, Berlin, 2003; ders.: Niedersachsen: Staatliche Repräsentanz in den Regionen. Funktion, Aufgaben und Organisation von „Regierungsbüros“, Berlin, 2004; ders.: Überprüfung der kommunalen Verwaltungsstrukturen im Saarland, Berlin, 2004; ders.: Förderung der interkommunalen Zusammenarbeit in Niedersachsen. Zwischenbericht, Berlin/Hannover, 2005; ders.: Reorganisation der Hauptstadtverwaltung. Funktional- und Verwaltungsstrukturreform in Berlin, Berlin, 2005; ders.: Modelle der Stadt-Umland-Organisation in der Bundesrepublik Deutschland. Vergleichsanalyse und Schlussfolgerungen für den Raum Saarbrücken, Berlin, 2005; ders.: Raumordnung und Landesentwicklung. Reformoptionen für ein tradiertes Politikfeld, Baden-Baden, 2006; ders.: Aufgabenkritik, Funktional- und Strukturreform in den Flächenländern. Das Beispiel Saarland, Baden-Baden, 2007; ders.: Kreisgröße und kommunales Ehrenamt, Berlin, 2008; ders.: Verwaltung erfolgreich modernisieren. Das Beispiel einer Kreisgebietsreform, Baden-Baden, 2008; Hesse, J.J./Götz, A.: Staatsreform in Deutschland - das Beispiel der Länder, Teil I in: ZSE, 1/4 (2003), 579-612, Teil II in ZSE, 2/1 (2004), 106-143; dies.: Systematische Aufgabenkritik in der nordrhein-westfälischen Landesverwaltung. Auswertung der Aufgabenerhebung und Ansatzpunkte für die 
Teile der Föderalismusreform sowie eine überfällige Aufgaben- und Organisationskritik auf Seiten des Bundes, gilt für diese Ebene und ihre Einrichtungen dem Grunde nach das Gleiche. Über die einbezogenen Träger dürfte das $S G B I I$ demnach auf die Konstitution und Leistungsfähigkeit des öffentlichen Sektors beträchtlichen Einfluss nehmen und für die betroffenen Gebietskörperschaften eine längerfristige Bewährungsprobe darstellen.

\section{Das Urteil des Bundesverfassungsgerichts und die Ergebnisse der § 6c-Evaluation: einseitige Diskussion im Vorfeld der politischen Entscheidung}

Nach dem Urteil des Bundesverfassungsgerichts zur Unzulässigkeit der Mischverwaltung in den ARGEn nach $\S 44 \mathrm{~b}$ SGB II und dem Abschluss der Wirkungsforschung zu den Trägermodellen gemäß $\S 6 \mathrm{c}$ SGB II steht der Gesetzgeber vor einer Entscheidung zur Organisation der Grundsicherung für Arbeitsuchende. Dabei ist zu vermeiden, dass die Abwägung zwischen Vor- und Nachteilen alternativer Trägerformen erneut (wie schon 2004) durch spezifische Organisationsinteressen und parteipolitische Orientierungen überlagert wird, funktionsanalytische Erkenntnisse hingegen zurücktreten. Die Überlegung, mit den ARGEn eine in mehrfacher Hinsicht dysfunktionale Konstruktion nachträglich über eine Verfassungsänderung zu legitimieren, lässt jedoch genau das befürchten. Problematisch erscheint hieran vor allem die unterlassene Würdigung praxisrelevanter wissenschaftlicher Erkenntnisse dies- wie jenseits der offiziellen Wirkungsforschung. Dies gilt umso mehr, als die Bewertung der Evaluationsergebnisse von der Datenbasis und von politischen Kriterien abhängig bleibt. Deshalb ist vor voreiligen Schlüssen zu warnen, zumal eine erweiterte empirische Basis belegt, dass für einseitige Trägerentscheidungen weder sachliche Gründe noch eine funktionale oder rechtliche Veranlassung bestehen. Vielmehr bieten sich Lösungen an, die Bundes- wie kommunalen Interessen, vor allem aber den Ansprüchen der Hilfebedürftigen, gerecht werden.

\footnotetext{
Neuordnung der Kompetenz- und Organisationsstrukturen, Berlin, 2004; dies.: Struktur- und Kommunalisierungsbenchmark. Systematischer Ländervergleich zur Aufbauorganisation und staatlichkommunalen Zuständigkeitsverteilung, Berlin, 2005; dies.: Kooperation statt Fusion? Interkommunale Zusammenarbeit in den Flächenländern, Baden-Baden, 2006; dies.: Für eine zukunftsfähige Arbeits- und Sozialverwaltung, a. a. O.; dies.: Gesetz zur Weiterentwicklung der Verwaltungsstrukturreform (VRWG) in Baden-Württemberg. Gutachterliche Stellungnahme, Berlin, 2008; dies.: Finanzieller Ertrag einer Kreisgebietsreform. Methode und Beispiel der Berechnung von Fusionsrenditen, Baden-Baden, 2008; dies.: Evaluation der Arbeit und Wirkungsweise der Niedersächsischen Regierungsvertretungen (2005-2008), Berlin, 2008.
} 


\section{Erfordernis einer erweiterten Perspektive - notwendiger Einbezug sozialpolitischer, funktionaler und staatspolitischer Kriterien}

Die aus der offiziellen (BMAS-)Evaluation abgeleitete These, in ARGEn würde besser in bedarfsdeckende Beschäftigung vermittelt und deshalb effizienter gearbeitet als in anderen Trägerformen, dürfte so nicht zu halten sein. Sie gründet mit einem einjährigen Zeitraum (2006/2007) auf einer entschieden zu schmalen und mit Unschärfen behafteten Datenbasis, die eine substantielle Betrachtung von Nachhaltigkeitseffekten ausschließt. Dies stellt nicht den statistischen Befund als solchen, aber seine Verallgemeinerungsfähigkeit in Frage. Zugleich werden jene Vorteile der Optionskommunen, die auch seitens der Wirkungsforschung im Auftrag des Bundes anerkannt werden, systematisch unterschätzt. Sie bestehen insbesondere in einer höheren Vermittlung in auch nicht vollständig bedarfsdeckende Beschäftigung sowie in einer besseren sozialen Stabilisierung der Hilfebedürftigen und ihres Umfelds. Hieran zeigt sich, dass die Bewertung des BMAS unverändert durch eine einseitige Vermittlungsorientierung geprägt ist, Beschäftigungsfähigkeit und erweiterte soziale Integration indes vernachlässigt werden. Dies erscheint nicht nur sozialpolitisch und volkswirtschaftlich fragwürdig, sondern geht auch an dem Bedarf einer zunehmend von schwerwiegenden Vermittlungshemmnissen betroffenen Klientel vorbei. Hinzukommt, dass die auf der Basis eng geführter Beurteilungsmaßstäbe hergeleiteten ARGE-Vorteile institutionelle Kontexte ausblenden. So sollte im Rahmen der Bundes-Evaluation erkennbar geworden sein, dass die Modellkonkurrenz zwischen ARGEn, Optionskommunen und getrennter Aufgabenwahrnehmung positive Wettbewerbseffekte in Form von Leistungsanreizen auslöste und schon allein deshalb einseitige Trägerentscheidungen nicht zu begründen sind. Die nachfolgend dargestellten Ergebnisse der SGB II-Evaluation des Internationalen Instituts für Staats- und Europawissenschaften (ISE) belegen für die Jahre 2005 bis 2008, dass mit Blick auf Klientel und Geschäftspolitik sowie Schnittstellen und soziale Integration Optionskommunen mit ihrer Problemnähe und hohen Flexibilität wesentliche Vorteile besitzen; sie eröffnen im Ergebnis die Chance zu einer sinnvollen Beantwortung der strittigen Trägerschaftsfrage.

\section{Klientel und Geschäftspolitik - strukturelle Hilfebedürftigkeit als Argument für dezentrale Problemnähe}

Auf der Basis einer dreimaligen flächendeckenden Befragung aller Landkreise und Optionsstädte sowie vertiefender Fallstudien in zahlreichen Optionskommu- 
nen und ARGEn stellte das ISE seit 2005 einen besonders hohen Bestand an schwer Vermittelbaren fest. Dieser Anteil hat sich bis heute deutlich vergrößert. Eine nachhaltige Integration von Hilfebedürftigen ist deshalb mehr denn je von intensiver Förderung und zielgruppenorientierter Betreuung abhängig. Allenfalls in strukturschwachen Regionen kommt einer überregionalen Vermittlung noch eine nennenswerte Bedeutung zu. Hinzutreten verstärkte Bemühungen um präventive Ansätze zur Vermeidung künftiger und der Verfestigung bestehender Hilfebedürftigkeit. In allen benannten Kategorien (individuelle Förderung, Zielgruppenorientierung, Prävention) schneiden die Optionskommunen besser als die anderen Trägerformen ab. Ihnen kommen ihre Flexibilität und Unabhängigkeit von der zentralen Organisation der Bundesagentur zugute. Beides gestattet eine deutlich problem- und klientelnähere Gestaltung von Abläufen und Eingliederungsstrategien. Insofern erweist sich der lokale Bezug als entscheidender Vorteil, während bundesweite Vermittlungssysteme weniger bedeutend erscheinen.

\section{Schnittstellen und soziale Integration - erneuter Vorteil einer dezentralen Ausrichtung und Vollzugsverantwortung in den Optionskommunen}

Nach Einschätzung aller vom ISE befragten Praktiker ist der erfolgreiche Vollzug des SGB II auf die Vernetzung mit parallelen Leistungssystemen und anderen öffentlichen Aufgaben angewiesen. Dabei spielen kommunale Zuständigkeiten in den Bereichen Soziales, Jugend, Schule, Gesundheit und Wirtschaft eine herausgehobene Rolle. Hinzutritt die bedarfsgerechte Erbringung flankierender Leistungen (Sucht- und Schuldnerberatung, psychosoziale Betreuung). Aufgrund der ISE-Erhebungen und kontinuierlicher Anschauung vor Ort lässt sich dokumentieren, dass die Optionskommunen über eine deutlich problemnähere Sicht verfügen und stärker als andere Träger um eine operative Berücksichtigung von Schnittstellen und sozialintegrativen Leistungen bemüht sind. Dies erklärt sich vor allem aus dem generell höheren Stellenwert, den die zugelassenen Träger der sozialen Stabilisierung von erwerbslosen Hilfebedürftigen und ihren Bedarfsgemeinschaften beimessen. Zwar finden sich anerkennenswerte Routinen auch in den ARGEn, doch stehen dort entsprechenden Ansätzen nach wie vor die Beteiligung zweier Träger und deren unterschiedliche Verwaltungskulturen entgegen. Hinzutritt die bereits angesprochene Fixierung der BA-Systeme auf den blo $\beta$ kurzfristigen Vermittlungserfolg. Zwar können die ARGEn eine bessere institutionelle Anbindung an sonstige Agenturleistungen geltend machen (insbesondere zum SGB III und SGB IX), doch bestehen in diesen Bereichen (mit Ausnahme vor allem des Arbeitgeberservice) entsprechende Kooperationen auch zwischen 
Optionskommunen und Agenturen; sie dokumentieren erneut eine hohe Kooperationsbereitschaft der zugelassenen Träger.

\section{Trägerentscheidung und Handlungssituation - eindeutiges Votum für kommunale Verantwortung und lokale Spielräume}

Im Ergebnis schätzen die Optionskommunen wie das ISE deren operative Handlungs- und Gestaltungsmöglichkeiten deutlich positiver als die der anderen Träger ein. Hinzutreten die benannten Leistungsanreize aus der Modellkonkurrenz. Hiervon profitieren dem eigenen Vernehmen nach auch die ARGEn, sofern die beteiligten Kommunen mit Verweis auf eine mögliche eigene Trägerschaft dezentrale Spielräume erstreiten. Jene ,Option der Option “ hängt selbstverständlich von der Glaubwürdigkeit des institutionellen Drohpotenzials ab. Dieses wirkte sich vor 2005 und zu Beginn des neuen Leistungssystems stärker als in den vergangenen zweieinhalb Jahren aus. Hier führten zuletzt Einflussnahmen des Bundes i. S. einer stärkeren Fach- und Regelsteuerung (Rahmenvereinbarung und sog. Rollenpapier als Beispiele) zu einer spürbaren Unzufriedenheit und Demotivierung der kommunalen Partner wie der operativ Verantwortlichen. Deshalb würde inzwischen eine Mehrheit der in ARGEn engagierten Landkreise bei einer erneuten Trägerentscheidung optieren. Ähnliches gilt für die Kommunen mit getrennter Aufgabenwahrnehmung. Interessant ist hierbei, dass der Vollzug eines Wechsels der Trägerform vor allem dann zeitnah in Erwägung gezogen würde, wenn dies nur befristet und (erneut) für eine begrenzte Zahl von Kommunen gelten sollte. Bei einer vollständigen, also dauerhaften und unkontingentierten Öffnung der Option hielten das deutlich weniger Kreise für unmittelbar erforderlich. Damit wird erkennbar, dass es den Betroffenen vor allem darum geht, mehr Spielräume für eine dezentrale Vollzugsgestaltung zu gewinnen; diese für den Integrationserfolg zentrale Handlungsbedingung ist in den ARGEn aufgrund ihrer bisherigen Konstruktion nicht in ausreichendem Maße gegeben.

\section{Institutioneller Nachteil der ARGEn als systemischer Vorteil der Option - Möglichkeit eines produktiven Kompromisses}

Summiert man die Evaluationsergebnisse des ISE und bezieht dabei - soweit bekannt - die Erkenntnisse der Wirkungsforschung des Bundes mit ein, lässt sich feststellen, dass über alle örtlichen Einrichtungen hinweg und in jeder Trägerkategorie sich sowohl gute als auch weniger leistungsstarke Fälle finden. Der Erfolg hängt vor allem von der örtlichen Organisationsgestaltung und Vollzugs- 
praxis ab. Hierfür wiederum bringen die Optionskommunen deutlich bessere Voraussetzungen mit. Strebt man darüber hinaus eine generelle Kennzeichnung der Trägermodelle an, so sind den ARGEn gewisse Stärken im Hinblick auf die stellenorientierte Vermittlung und die Vernetzung mit dem SGB III-Bereich zu attestieren. Demgegenüber verfügen die Optionskommunen aber über die benannten systemischen Organisationsvorteile und können klare Erfolge bei der sozialintegrativen Einbettung der Integrationstätigkeit unter Einschluss präventiver Ansätze nachweisen. Fälle mit getrennter Aufgabenwahrnehmung wiederum erlauben eine separate Optimierung des Vollzugs von kommunalen und Agenturaufgaben, leiden aber entscheidend unter Schnittstellen und einem fehlenden gesamthaften Integrationsansatz. Mit Blick auf die auch bei veränderter Arbeitsmarktlage schwierigen Klientelanforderungen führt dies letztlich zu einem $V o$ tum für die kommunale Trägerschaft, insbesondere dann, wenn man eine nachhaltige Integration und Stabilisierung von Hilfebedürftigen als wenigstens ebenso wichtig wie den bloßen Vermittlungserfolg einschätzt.

\section{Eine zukunftsfähige Kompromissvariante: Öffnung der Option und Fortschreibung des Modellwettbewerbs}

In der Konsequenz muss aber auch dieser Befund nicht notwendiger Weise zu einer flächendeckenden Zuständigkeit der Kommunen und (auf verfassungsgemäßem Wege) der Länder führen. Vielmehr belegt gerade das differenzierte Wahlverhalten der Kreise bei einer erneuten Optionsmöglichkeit, dass es vor allem um die dezentralen Entscheidungsspielräume und eine erweiterte Kooperationsfreiheit geht. Insofern erschiene es ausreichend, eine vollständige und dauerhafte Option zu gestatten und die ARGEn im SGB II nicht mehr als verbindliche Organisationsform auszuweisen. Damit würde eine ohnehin unter rechtssystematischen Gesichtspunkten wenig überzeugende Verfassungsänderung obsolet und ließe sich der im Ergebnis positive Modellwettbewerb fortschreiben. Legitime Berichts- und Prüfansprüche des Bundes wären wie heute über bestehende Kontrollverfahren abzusichern. Die inhaltliche Steuerung des SGB II und seiner aktiven Hilfen scheinen über das SGB II hinreichend gewährleistet, ließen sich aber - wenn auch in eng zu setzenden Grenzen - erforderlichenfalls über untergesetzliche jährliche Verordnungen für alle Träger präzisieren. Schließlich sollte erwogen werden, Softwarestandards sowie die Festlegung von Erfolgsindikatoren und Statistikpflichten stärker als bislang abzustimmen und die kommunalen Träger hieran angemessen zu beteiligen. 


\section{Modellvergleich: Beurteilungskriterien, Situation der drei Trägermodelle, Performanz von ARGEn und Optionskommunen}

Im Ergebnis der seitens des ISE vorgelegten Untersuchungen kann ein zusammenfassender Vergleich der Performanz der gegenwärtigen Trägermodelle vorgelegt werden. Als Ergebnis des Evaluationsansatzes steht dabei die örtliche Handlungs- und Gestaltungsfähigkeit als zentraler Erfolgsfaktor im Mittelpunkt. Dies ist sowohl für Optionskommunen als auch für ARGEn im Hinblick auf deren spezifische Stärken und Schwächen nachweisbar. Hinzutritt die gesonderte Situation getrennter Aufgabenwahrnehmung, der im Nachgang zum Urteil des Bundesverfassungsgerichts (zumindest zwischenzeitlich) erhöhte Aufmerksamkeit zukam. Die Bedeutung der angelegten Kriterien und deren politische Dimension verweist auf die Notwendigkeit, Strategien und Methoden der Erfolgsmessung bei der Dokumentation des Modellvergleichs mitzudenken. Schließlich werden in einer gesamthaften Bewertung die wesentlichen Gesichtspunkte der zu bilanzierenden Systemkonkurrenz mögliche wie erwartbare Unterschiede zur offiziellen Evaluation des Bundes nach § 6c SGB II ausgewiesen.

\section{1. Örtliche Handlungs- und Gestaltungsfähigkeit als Erfolgsfaktor}

Wie die Einzelergebnisse der Untersuchung dokumentieren, ${ }^{4}$ erweist sich die $l o-$ kale Flexibilität und Reaktionsfähigkeit als entscheidende Voraussetzung für einen effektiven Vollzug des SGB II. Dies folgt unmittelbar aus den Eigenschaften der zu betreuenden Klientel, für das eine allein auf den Ersten Arbeitsmarkt hin orientierte Vermittlungstätigkeit ausscheidet. Dabei sollten maßgeschneiderte und meist niederschwellige Beschäftigungsangebote in örtlichen und regionalen Kontexten gefunden werden, was sich mit erhöhten Anforderungen an eine sozialintegrative Flankierung und bildungsbezogene Absicherung der Hilfen verbindet. Beides führt vor allem im aktiven Bereich zu einer zunehmenden Spezialisierung und Individualisierung der gewährten Leistungen (hinzutritt die notwendige Verknüpfung mit anderen kommunalen Aufgaben - etwa in der Jugendhilfe und im Bildungswesen) und erfordert einen qualifizierten Personal-

4 Hesse, J.J./Götz, A.: Evaluation der Aufgabenträgerschaft nach dem SGB II 2005-2008. Abschlussbericht, Berlin, 2008; dies.: Evaluation der Aufgabenträgerschaft nach dem SGB II in Baden-Württemberg 2005-2008. Abschlussbericht, Berlin, 2008. Eine zusammenfassende Publikation erfolgt im Rahmen der Schriftenreihe „Staatsreform in Deutschland und Europa. Der öffentliche Sektor im nationalen und internationalen Vergleich“ im Nomos-Verlag (Frühjahr 2009). 
einsatz, mit dem auf die gebotene Differenzierung angemessen reagiert werden kann.

\section{Systematischer Gestaltungsvorteil der Optionskommunen}

Zudem konnte nachgewiesen werden, dass alle Träger und örtlichen Einrichtungen diesen Rahmenbedingungen in der beschriebenen Weise zu entsprechen suchen. Zugleich verdeutlichen die gegebenen und durchaus systematischen Unterschiede die nicht identischen Möglichkeiten, dies auch in vollem Umfang zu verwirklichen. Insofern erscheinen Organisation und Organisationshoheit als wesentliche Faktoren, um die Grundsicherung für Arbeitsuchende wirkungsvoll durchzuführen, wobei der dezentralen Reaktions- und Anpassungsfähigkeit eine erhöhte Bedeutung zukommt. Diese wiederum ist ausweislich der hier präsentierten Ergebnisse in Optionskommunen erkennbar höher ausgeprägt, was sich an der Ausgestaltung der Organisationsstrukturen und Abläufe, einer eigenständigen (und flexibleren) Personal- und Ressourcenbewirtschaftung, den Maßnahmenportfolios und zielgruppenbezogenen Instrumenten, der nach wie vor intensiveren Nutzung von Schnittstellen und Kooperationspotenzialen sowie dem ebenfalls stärkeren Einbezug von flankierenden und sozialintegrativen Leistungen nachvollziehen lässt.

\section{Institutionell bedingte Nachteile der ARGEn}

Demgegenüber verweisen die operativen Gegebenheiten in den ARGEn nicht auf eine grundsätzlich geringere Performanz, aber auf eine deutlich eingeschränkte Fähigkeit, individuelle Organisations- und Geschäftslösungen zu erarbeiten. Verantwortlich sind dafür zum einen die Einflussnahmen der Bundesagentur, die aus ihrer Trägerfunktion und den Verpflichtungen gegenüber dem Bund abgeleitet werden, zum anderen die Restriktionen, die sich aus der Kooperation zweier Träger ergeben. Ganz unmittelbar folgen hieraus häufigere „Kompromiss“-Lösungen, die naturgemäß nicht allein den operativen Anforderungen genügen können, sondern auch die Möglichkeiten der beteiligten Träger berücksichtigen müssen (etwa im Hinblick auf die Personalwirtschaft). Ungeachtet etwaiger Konflikte zwischen Agenturen und Kreisen (bzw. Städten) führt dies zu einer eklatanten Benachteilung der ARGE-Geschäftsführungen und ihrer Verantwortung für das operative Geschäft (gegenüber ihren Pendants in Optionskommunen). Am sichtbarsten wird dies anhand der beklagten (und in der lokalen Wahrnehmung auch dominanten) Software- und Personalentwicklungsprobleme; hin- 
zutreten die bereits angesprochenen und nach Darstellung der örtlichen Akteure über die vergangenen vier Jahre erkennbar zunehmenden Einflussnahmen der Bundesagentur. Hieraus resultieren aufgrund von Schnittstellen, Steuerungskonflikten und Unterschieden in der Verwaltungskultur höhere ,Abnutzungserscheinungen " beim Leitungs- und operativen Personal der ARGEn, wobei Spannungen vor allem in solchen Einrichtungen evident sind, die von kommunalen Geschäftsführern geleitet werden. Als Konsequenz strebt die überwiegende und eine zudem wachsende Zahl der Beteiligten eine organisatorische oder sogar trägerbezogene Vereinheitlichung der Aufgabenverantwortung an. Seitens der ARGEn spiegelt sich dies in der Forderung nach einer rechtlichen Verselbständigung und eigenen Dienstherrenschaft (zwecks Einstellung eigenen Personals und der Gestaltung einer souveränen Personalpolitik) wider. Die an den ARGEn beteiligten Kommunen wiederum erkennen in zunehmendem Maße, dass nicht nur die Besetzung der operativ verantwortlichen Position des Geschäftsführers, sondern auch die Verbindung mit dem Vorsitz in der Trägerversammlung erforderlich ist, um eigene Interessen zu wahren und einen vernetzten Vollzug herzustellen. Demgegenüber lehnt die große Mehrheit eine operative Trennung der Trägerkompetenzen ab, und zwar sowohl in einer grundsätzlichen, der separaten Aufgabenwahrnehmung nachempfunden Form als auch innerhalb der ARGEn, wie dies vom Bund wiederholt und mit dem sog. „Rollenpapier“ auch bereits vor der Karlsruher Entscheidung in die Diskussion gebracht wurde. Insofern richtet sich die kommunale Kritik nicht gegen einen einheitlichen und nur i.T. gegen einen gemeinsamen oder abgestimmten Vollzug, sondern vor allem gegen die derzeitige Ausgestaltung der Mischverwaltung und gegebenen Kompetenzverteilung.

\section{Getrennte Aufgabenwahrnehmung als Ausweichlösung mit erheblichen Vollzugsrisiken}

Gerade mit Blick auf den weithin als vordringlich betrachteten Funktionszusammenhang zwischen den im SGB II auf Bund und Kommunen aufgeteilten Zuständigkeiten erscheint die getrennte Aufgabenwahrnehmung allenfalls vordergründig als Alternative zu einer konflikthaften ARGE-Bildung oder der als zu riskant eingeschätzten Optionsentscheidung. In nahezu allen vom ISE erhobenen Fragekomplexen, in die entsprechende Kommunen einbezogenen werden konnten, wurde 2007 und 2008 eine im Vergleich zu ARGEn und Optionskommunen verminderte Abstimmungsintensität festgestellt (etwa bei Schnittstellen und flankierenden Leistungen). Damit aber finden sich zwei wesentliche Anforderungen 
an die Grundsicherung für Arbeitsuchende in Frage gestellt: die angestrebte Hilfegewährung aus einer Hand sowie die Vernetzung von nicht in gemeinsamer Verantwortung befindlichen Leistungen (insbesondere aus dem kommunalen Bereich). Lediglich die unmittelbaren Anknüpfungspunkte einer abgestimmten Berechnung und Auszahlung des Arbeitslosengeldes mit seinen bundesseitigen und kommunalen Bestandteilen konnten bislang vergleichsweise pragmatisch ausgestaltet werden. Dabei allerdings verbleiben den Kommunen im Regelfall nur der Nachvollzug und die Umsetzung der seitens der Agentur ermittelten Leistungsansprüche, da es anderenfalls zu einem widersprüchlichen Verwaltungshandeln der Träger käme. Eine darüber hinausgehende Kooperation, die organisatorische Verflechtungsprobleme der ARGEn (etwa im Personalbereich) vermeidet, jedoch wie in diesen oder den Optionskommunen zu einer ähnlichen Kompetenzbündelung gelangt, ließ sich nicht nachweisen. Diese Defizite werden auch nicht dadurch relativiert, dass nach Darstellung von BMAS und BA die Jobcenter bzw. Agenturen bei separatem Vollzug vergleichsweise gute Vermittlungszahlen aufweisen. Denn erneut ist diese Bewertung Ausdruck einer Zielfixierung ohne hinreichende Berücksichtigung der sozialintegrativen Dimension des SGB II.

\section{Bedeutung und politische Dimension der Bewertungskriterien}

Die einseitige Programmierung des SGB II erschwert grundsätzlich eine Messung der Leistungsfähigkeit, wobei die in hohem Maße kontextabhängige Vermittlung als eher zufälliges Kriterium erscheint, vergleicht man dies mit dem Anspruch, nicht nur die Arbeitslosenzahlen, sondern vor allem die gesamtgesellschaftlichen Lasten und Folgewirkungen der Langzeitarbeitslosigkeit zu senken. Die Entscheidung der Trägerfrage droht deshalb bei unveränderten Rahmenbedingungen erneut zu einem reinen Politikum zu werden, dessen Beantwortung sich nur sehr bedingt mit belastbaren Evaluationsergebnissen absichern lässt, wenn deren adäquate Interpretation eine normative $b z w$. ordnungspolitische Prioritätensetzung voraussetzt. Dabei geht es erneut vor allem um die Frage, ob mittels des SGB II zunächst und vor allem die Zahl der Erwerbslosen und Anspruchsberechtigen reduziert werden soll (so die Schwerpunktsetzung bislang) oder ob zumindest zu gleichen Teilen die Ursachen, also die im Klientel selbst angelegten Voraussetzungen dauerhafter Hilfebedürftigkeit und deren Ausmaß zu bekämpfen sind. Ersteres würde die bereits festgestellten Tendenzen einer zunehmenden Kundenselektion befördern und dürfte sich mit Blick auf die gegebene BA-Anbindung eher zugunsten der ARGEn (insbesondere in strukturschwa- 
chen Regionen, großen Städten und den neuen Ländern) auswirken, sofern es den Optionskommunen (vor allem in Ländern mit besserer Arbeitsmarktlage und anteilig höherer Klientel mit verfestigten Vermittlungshemmnissen) nicht gelingt, durch ortsgebundene und maßgeschneiderte Angebote die ggf. besseren überregionalen Zugänge der ARGEn zu kompensieren. Umgekehrt müssten die Optionskommunen bei einer sozialintegrativ ausgerichteten Anpassung bzw. Konkretisierung des Handlungsauftrags nach dem SGB II sehr viel mehr als die ARGEn dazu in der Lage sein, die Eingliederung von Hilfebedürftigen und deren Angehörigen in mehreren Stufen und dabei sehr viel breiter anzulegen, selbst wenn dies weniger häufig zu einer kurzfristigen Vermittlung in eine bedarfsdeckende Beschäftigung auf dem Ersten Arbeitsmarkt führen würde.

\section{Daten, Strategien und Methoden der Erfolgsmessung}

Die Erhebung von Daten zur Sozioökonomie im Allgemeinen sowie zur Sozialund Arbeitsmarktpolitik im Besonderen genießt einen hohen politischen Stellenwert. Deshalb spiegeln sich (jenseits konkreter Steuerungsbedarfe) in Auseinandersetzungen um Zahlen und Messkonzepte regelmäßig ordnungspolitische Orientierungen wider, was naturgemäß nicht den unmittelbaren Interessen operativer Einrichtungen entspricht. Insofern erweist es sich als außerordentlich schwierig, im Kontext des SGB II Prämissen für eine möglichst bedarfsorientierte und objektive Statistik zu formulieren, die dann wiederum die Basis für eine aussagefähige Erfolgsmessung bilden könnten.

Dies gilt umso mehr, als der bisherige Verlauf des neuen Leistungssystems durch schwerwiegende Datenprobleme gekennzeichnet war. Ausgehend von dem Statistikskandal bei der Bundesanstalt für Arbeit, der den Auslöser der sog. „Hartz“Reformen bildete, kam es im Zuge der Einführung des SGB II 2005 und 2006 zu einem regelrechten Fallzahlenschock, der auf unterschätzte Problemlagen und Bedarfe, leistungsrechtlich bedingte Fehlanreize und eine problematische Wirtschaftslage zurückzuführen war. Hinzutraten operative Probleme beim Aufbau von Melde-, Statistik- und EDV-Systemen, die bis in das Jahr 2007 vor allem die Optionskommunen, mit Blick auf die Leistungssoftware A2LL aber ebenso die ARGEn betrafen. Zugleich bestehen bis heute bei Kommunen und örtlichen Einrichtungen erhebliche Zweifel, ob die verwandten Indikatoren und Kennzahlen einem zu schematischen Messkonzept folgen und damit letztlich an den Erfordernissen des Arbeitsmarktes vorbeigehen. Dies verband sich von Beginn an mit beträchtlichen Konflikten um problemadäquate Messgrößen wie Arbeitslosigkeit, Zahl der Arbeitsuchenden, Quantität der Bedarfsgemeinschaften und 
erwerbsfähigen Hilfebedürftigen. Im Ergebnis führte dies zu divergenten Messund Evaluationsstrategien der kommunalen und agenturseitigen Grundsicherungsträger, die erneut den Widerstreit zwischen primärer Vermittlungs- und erweiterter Integrationsorientierung dokumentieren.

Im Rahmen der ISE-Fallstudien wurden auch zu diesen Themenstellungen 20 Grundsicherungsträger in den vergangenen vier Jahren regelmäßig befragt. Dabei wurde erkennbar, dass es dem Gesetzesauftrag folgend in allen Fällen zu einer prioritären Erfassung und handlungsleitenden Bewertung von Integrationen in den Ersten Arbeitsmarkt kommt. Ein weiteres (gemeinsames) Interesse besteht in der Entwicklung der Höhe passiver Leistungen, was aus Sicht der Städte und Kreise insbesondere die Ausgaben für die Kosten der Unterkunft betrifft. Spezifische, aber nach wie vor modellunabhängige Prioritäten bestehen beim Nachvollzug geeigneter Indikatoren für die Erfassung von Erfolgen in der Arbeit mit schwer Vermittelbaren (etwa über die regelmäßige Beachtung der Senkung der Zahl von Arbeitslosen mit einem Bezug von länger als 24 Monaten). Hinzutreten diverse ,händisch" durchgeführte Analysen - etwa zur Rolle der jeweiligen Einrichtung im Integrationsprozess, also zur Frage, ob sich Erwerbslose vor allem durch Eigeninitiative oder durch wesentlichen Beitrag des Grundsicherungsträgers integrieren konnten. Ähnliches gilt für die Messung des spezifischen Maßnahmenerfolgs anhand von Fallauswertungen oder durch Zählung von Abbrecherquoten.

Unterschiede zwischen den Trägermodellen bestehen indes vor allem darin, dass die Optionskommunen intern wie auch gegenüber der Kommunalpolitik verstärkt übergreifende Quoten zur Entwicklung der Zahlen von Bedarfsgemeinschaften, erwerbsfähigen Hilfebedürftigen und Leistungsbeziehern nutzbar machen. Außerdem achten zugelassene Träger offenbar häufiger auf erfasste Fortschritte im Integrationsstatus, obgleich man auch hier Messprobleme konzediert und allenfalls von einer „endogenen“ Objektivität ohne weitergehende Vergleichbarkeit ausgehen kann, sofern etwa die Kundenklassifikation nach Arbeitsmarktnähe unmittelbare Verteilfunktionen im internen Geschäftsgang erfüllt. Eine komparative und damit verallgemeinerungsfähige Bewertungsbasis steht hierbei auch für die Gruppe der Optionskommunen nicht zur Verfügung, zumal die einzelnen Einrichtungen sehr unterschiedlichen Systemen folgen. Zwar scheinen auch immer mehr ARGEn diesem Aspekt - nicht zuletzt im Gefolge der VerBis-Einführung - höhere Bedeutung beizumessen, doch werden die hierüber generierbaren Informationen derzeit nach Angaben der vor Ort befragten Akteure kaum oder sehr wenig genutzt. 
Demgegenüber greift man in den ARGEn häufiger auf die in den BA-Controlling und Benchmarkberichten enthaltenen Größen zurück, obgleich vielerorts eine erhebliche Skepsis gegenüber der zugrunde liegenden Datenbasis besteht. Gleichwohl erweist sich die Orientierung an diesen Indikatoren meist als alternativlos, da seitens der Agentur entsprechende Größen im Zielvereinbarungsprozess und in den daran ansetzenden „Nachhalte“-Verfahren dezidiert abgefragt werden. Damit einhergeht eine höhere Aufmerksamkeit für betriebswirtschaftliche Input-Faktoren, wie etwa die Verwaltungskosten je erwerbsfähigem Hilfebedürftigen. Hinzutreten prozessbezogene Teildaten, wie etwa Zeiträume zwischen Erstberatung und Abschluss einer Eingliederungsvereinbarung, u. ä.m.

Auf einhellige Kritik stoßen dagegen die insgesamt noch nicht hinreichenden Aussagen zu einer belastbaren Verbleibstatistik, die neben den unmittelbaren Vermittlungserfolgen auch deren Nachhaltigkeit dokumentiert. Problematisiert werden zudem die seitens der BA verwandten Vergleichscluster, da man hierüber die notwendige Berücksichtigung institutioneller, sozialer wie ökonomischer Rahmenbedingungen und Unterschiede für nicht adäquat abgebildet hält. Freilich sind ähnliche Defizite auch für kommunale Eigenlösungen kennzeichnend, sofern etwa Vergleichsringe aufgrund fehlender Beteiligung und Vollständigkeit weder für die Betroffenen selbst noch für die politische Auseinandersetzung nutzbar sind.

Im Ergebnis führt zu der Einschätzung, dass sich jenseits der pflichtigen Statistikmeldungen nach dem SGB II eine einheitliche Bewertungs- und Steuerungspraxis auf der Basis differenzierter Vollzugs- und Erfolgsdaten noch nicht herausgebildet hat. Daraus wiederum folgt, dass die Bewertung dessen, was eine effektive Aufgabenwahrnehmung nach dem SGB II ausmacht, ebenfalls erheblich differiert. In der Mehrzahl konzentriert sich dieser Unterschied im Vergleich von ARGEn und Optionskommunen auf den Widerstreit zwischen sozialintegrativer und vermittlungsorientierter Ausrichtung, wobei die Arbeitsgemeinschaften die letztgenannte Perspektive vor allem aufgrund jener Ziel- und Controllingsysteme wählen, denen sie im Rahmen der Weisungen und Vorgaben der Bundesagentur und des BMAS unterliegen. Befragt nach den Erfordernissen einer umfeldorientierten und stabilisierenden präventiven Prioritätensetzung schätzen ihre Vertreter diese nicht grundsätzlich geringer ein, als das für Optionskommunen festzustellen ist (Quelle: ISE-Erhebungen 2006 bis 2008). Insofern sind es in ihrem Fall vor allem die institutionellen Rahmenbedingungen, konkret vor allem die SGB III-zentrierte Steuerungs- und Handlungslogik der Bundesagentur, die ihre gegenwärtigen Vorgehensweisen prägen, während die Optionskommunen 
diesbezüglich bereits heute wesentlich stärker von ihren Freiheiten Gebrauch machen.

\section{Bewertung}

Auf der Basis der vierjährigen Evaluation gelangen die Autoren zu der folgenden Beurteilung der von ihnen untersuchten Trägermodelle. Dabei ist allerdings zu betonen, dass die dargestellten Ergebnisse keinen direkten statistischen Zusammenhang mit den Resultaten und der Performanz der Grundsicherungsträger enthalten und für diese Fragen deshalb auf qualitative, analytische und plausibilitätsgestützte Erwägungen zurückgegriffen werden muss. Hieraus wiederum ergibt sich freilich kein Nachteil gegenüber der $\S 6 c-E v a l u a t i o n ~ i m ~ A u f t r a g ~ d e s$ Bundes, die solche quantitativen Aussagen zu treffen sucht. Denn auch diese Untersuchung unterliegt den unterschiedlichen Kriterien und ordnungspolitischen Orientierungen. Vor allem aber muss die hierfür genutzte Datenbasis als außerordentlich schmal eingeschätzt werden. So waren sowohl in ARGEn als auch in Optionskommunen bis 2006/2007 unverändert Konsolidierungsprozesse $z u$ verzeichnen und kann deshalb überhaupt erst für das Jahr 2007 von einer weithin routinisierten Aufgabenerledigung vor Ort ausgegangen werden. Gerade den zugelassenen kommunalen Trägern war diese Zeit einzuräumen, um ihnen den Aufbau jener Kapazitäten und Routinen zu ermöglichen (etwa im Bereich der Stellenvermittlung und des Arbeitgeberservice sowie bei Controlling- und Softwaresystemen), auf die man innerhalb der ARGEn durch die Beteiligung der Bundesagentur sofort zurückgreifen konnte. Folglich müsste man schon aus methodischen Gründen die Jahre 2007 und 2008 abwarten und in die Evaluation vollständig miteinbeziehen, um die benannten etwaigen Verzerrungen zu nivellieren. Vor diesem Hintergrund lassen sich nach den Erkenntnissen der ISEEvaluation die drei Trägermodelle wie folgt kennzeichnen:

- Die ARGEn weisen erhebliche Systemdefizite vor allem im organisatorischen und personalwirtschaftlichen Bereich auf, womit sie sich in einer zentralen Handlungsressource - der dezentralen Ausgestaltung von Integrationsund Betreuungsprozessen - deutlich eingeschränkt sehen. Dem stehen gewisse Stärken im Hinblick auf stellenorientierte Vermittlungsroutinen und die Vernetzung mit dem SGB III gegenüber.

- Die Optionskommunen genießen - allerdings gleichsam diametral entgegen gesetzt - ähnliche organisatorische Vorteile aufgrund einer einheitlichen Personal- und Ressourcenbewirtschaftung sowie der erheblichen Freiheits- 
grade bei der Konzipierung von Aufbau- und Ablaufstrukturen. Hinzutreten klare Erfolge bei der sozialintegrativen Einbettung der Integrationstätigkeit, was (weiterhin) zunehmend und stärker als bei anderen Trägermodellen präventive Ansätze einschließt.

- Fälle mit getrennter Aufgabenwahrnehmung können zwar auf eine separate Optimierung der jeweiligen Verantwortlichkeiten verweisen, doch unterliegt der Vollzug von Agentur- und kommunalen Aufgaben ungelösten Schnittstellenproblemen, kommt es in der Regel zu einer schlechteren Einbindung flankierender Leistungen, bleiben die kommunalen Einwirkungsmöglichkeiten auf die Geschäftspolitik und damit auch die Steuerungspotenziale (etwa hinsichtlich der Kosten der Unterkunft) tatsächlich gering und findet die Formulierung eines gesamthaften Integrationsansatzes im Kontext arbeitsmarkt- wie sozialpolitischer Orientierungen nicht statt.

Stellt man dem nun die Ergebnisse der offiziellen $\$ 6 c$-Evaluation gegenüber, so ergibt sich daraus im Einzelnen kein grundsätzlicher Widerspruch zu den vorgetragenen Einschätzungen. Vielmehr wird erneut deutlich, dass die Bewertung des Modellwettbewerbs nahezu ausschließlich von den angelegten Maßstäben abhängig ist, vor allem, wenn man berücksichtigt, dass es selbst bei statistisch nachweisbaren Unterschieden immer noch eine relevante Zahl von good oder best practice-Fällen in jeder Vergleichsgruppe gibt. Erwartbar sind hierbei seitens der Bundes-Evaluation etwa die folgenden Aussagen:

- In den ARGEn und in den Jobcentern bei getrennter Aufgabenwahrnehmung kommt es zu einer zielgemäßen Konzentration auf die stellenorientierte Vermittlung sowie in der Summe (monetär) zu besseren Ergebnissen bei der Absenkung der Hilfebedürftigkeit und damit auch im Hinblick auf die fiskalische Effizienz.

- Die Optionskommunen setzen Schwerpunkte in der Bewerberorientierung (fördernder Ansatz) mit größeren Fortschritten bei der Hebung der Beschäftigungsfähigkeit und mehr Erfolg bei der Vermittlung in eine (auch) nicht bedarfsdeckende Beschäftigung.

Berücksichtigt man die eher begrenzte Signifikanz der 6c-Ergebnisse sowie die Unterschiede bei der Auswahl von Bewertungsmaßstäben, ist für die nach der Karlsruher Entscheidung notwendig gewordene Reform der Trägerstruktur eine parteipolitisch geprägte Entscheidung erneut nicht unwahrscheinlich. Umso mehr bedarf es neben den fachspezifischen auch weitergehender Gesichtspunkte, die ggf. dazu beitragen können, den anstehenden Prozess zu versachlichen und seine Ergebnisse auf eine breitere Grundlage zu stellen. Hierbei erscheint das 
BVerfG-Urteil insoweit als hilfreich, als es auf die Bedeutung staats- und verfassungspolitischer Erwägungen im Zusammenhang mit Themen der Föderalismusreform hingewiesen und somit wesentliche Anknüpfungspunkte benannt hat. Danach sollte es bei der Organisationsgestaltung des mit Abstand größten sozialen Leistungssystems des Landes darauf ankommen,

- die Grundsätze des subsidiären Verwaltungsföderalismus einzuhalten,

- koordinative und kooperative Verfahren bei gleichzeitig entflochtenen Kompetenzen zu stärken,

- neue effizienz- wie effektivitätsfördernde Rahmenbedingungen und Anreizstrukturen zu fördern (Stichwort Wettbewerbsföderalismus) sowie

- Nachhaltigkeitsgesichtspunkte auch im institutionellen wie sozialpolitischen Bereich zu beachten.

Als Fazit dieser Betrachtungen lässt sich mithin festhalten, dass die Option und damit eine weitgehende kommunale Vollzugsverantwortung im Lichte der in diesem Bericht dokumentierten Evaluations-Ergebnisse und unter Berücksichtigung der vorgenannten Kriterien eine Trägerform mit deutlich systemischen Vorteilen darstellt und somit als Modell der ARGE und einer getrennten Aufgabenwahrnehmung dauerhaft überlegen sein sollte.

\section{Schlussfolgerungen: Gesamtbilanz und Handlungsoptionen}

Die im Rahmen dieses Berichts dokumentierte Leistungsfähigkeit/Performanz der Trägerstrukturen im SGB II führt im Lichte des Karlsruher Urteils zu einer Reihe von Handlungsoptionen, die die materiellen und funktionalen Erfordernisse des neuen Leistungssystems aufnehmen. Diese Gestaltungsvarianten suchen die institutionell möglichen und derzeit auch politisch diskutierten Lösungsansätze zu systematisieren, wobei im Folgenden die Grundfrage nach einer einheitlichen oder (weiterhin) geteilten Trägerschaft des SGB II den Ausgangspunkt bildet. Dies schließt drei bislang eher hypothetische, jedoch in der gegenwärtigen Diskussion durchaus vertretene Szenarien ein, die sich mit einer Bundesauftragsverwaltung und/oder einem veränderten Leistungsrecht verbinden. Eine gesamthafte Dokumentation der kommunalen Präferenzen erfolgt am Ende dieses Kapitels.

\section{Materielle und funktionale Erfordernisse des SGB II}

Eine zentrale gesetzliche Vorgabe der Grundsicherung für Arbeitsuchende besteht in der notwendiger Weise personengebundenen Betreuung und Aktivierung 
von Langzeitarbeitslosen, deren aktive und passive Leistungen aus einer Hand erbracht werden sollten. Auch wenn sich der dabei erforderliche sozialintegrative Ansatz des SGB II in dem Hinweis erschöpft, dass Maßnahmen zum Erhalt der Erwerbsfähigkeit bzw. zur Vermeidung von Hilfebedürftigkeit ergriffen werden sollen, erscheint von daher die individuelle und langfristige Betreuung in weiten Teilen problembehafteter Zielgruppen (Dauerarbeitslosigkeit verbunden mit multiplen Vermittlungs- und sozialstrukturellen Integrationshemmnissen) mindestens ebenso wichtig wie die Optimierung der reinen Vermittlungstätigkeit. Die Verbesserung der Beschäftigungsfähigkeit sowie soziale Stabilisierung und Integration bilden somit zu Recht wesentliche Gesichtspunkte für die Beurteilung der Effektivität des neuen Leistungssystems. Hierbei wiederum kommt dem Einbezug unterschiedlichster flankierender Instrumente sowie dem Vollzug im Kontext und lokalen Netzwerk sozialer Daseinsvorsorge eine besondere Bedeutung zu. Die entsprechend personalintensiven Dienst- und Transferleistungen sind gerade unter erschwerten Klientelbedingungen von klaren Zielen und Aufträgen abhängig; sie erfordern zudem vor Ort störungsfreie Abläufe, klare und individuell gestaltbare Ziele sowie eine erhöhte Mitarbeitermotivation. Deshalb ist der Vollzug des SGB II weit mehr als viele andere öffentliche Aufgaben von einer funktionsadäquaten Organisation auf der Basis zusammenhängender, möglichst einheitlicher Trägerzuständigkeiten abhängig.

\section{Optionen nach dem BVerfG-Urteil}

Für die Weiterentwicklung des SGB II nach der Karlsruher Entscheidung gilt es einige grundlegende Fragen zu beantworten. Hierzu zählt zunächst die nach der Trägerschaft i.e.S., ob also die Grundsicherung für Arbeitsuchende weiterhin durch mehrere (Bund und Kommunen sowie indirekt die Länder) oder künftig nur noch durch einen Träger (Bund oder Kommunen bzw. Länder) wahrgenommen werden soll. Bei einer alleinigen Bundesträgerschaft bestünde ferner die (ergänzende) Möglichkeit des Aufgabenausgleichs zugunsten der Kommunen und Länder über eine Gewichtsverlagerung zwischen SGB II und SGB XII auf der Basis angepasster Erwerbsfähigkeitsregelungen. Komplexer stellt sich demgegenüber die Situation bei einer geteilten Trägerschaft dar. Hier wäre zunächst zu klären, ob es nur noch ein Modell (etwa eine Kooperation zwischen Bund und Kommunen, getrennten Vollzug oder die kommunale Durchführung) oder weiterhin verschiedene Varianten nebeneinander geben kann (Optionskommunen und ARGEn als Beispiel). Hinzutritt die Frage, wie eine Trägerform ausgestaltet wird, in der Bund und Kommunen erneut kooperativ zusammenwirken sollen. 
Des Weiteren muss die Zukunft und der Umgang mit dem Optionsmodell geklärt werden, wofür letztlich drei Alternativen bestehen: die bloße Fortschreibung der bestehenden zugelassenen Träger, die zeitlich befristete und/oder zahlenmäßig beschränkte Öffnung oder aber die vollständige, also weder terminierte noch kontingentierte Ermöglichung kommunaler Verantwortungsübernahme. Schließlich kämen Zwischenvarianten in Betracht, wie etwa die operative Vereinheitlichung des Vollzugs über eine vollständige oder teilweise Auftragsverwaltung des Bundes; denkbar wäre ferner eine Entflechtung des Leistungsbereichs durch eine Abkoppelung der Wohn- und Unterkunftskosten im Rahmen einer eigenständigen vom ALG II unabhängigen Transferleistung.

\section{Möglichkeit und Zuordnung einer einheitlichen Trägerschaft}

Befragt nach der künftigen Kompetenzordnung im SGB II lehnen neun von zehn Kommunen die bestehende geteilte Trägerschaft $a b$ und zwar unabhängig von der heutigen Trägerform (Quelle: ISE-Erhebung 2008). Zwar hält man unter den gegebenen Umständen eine Kooperation und Abstimmung zwischen Bund, Kommunen und Ländern für unverzichtbar, doch leitet sich dieses Erfordernis zu einem wesentlichen Teil aus den gesetzlichen Zuständigkeiten ab. Hinzutritt die Überzeugung, dass man den wünschenswerten Kompetenztransfer zwischen Bund und Kommunen auch auf dem Wege loser Kooperationsformen realisieren könne. Deshalb plädieren $95 \%$ der befragten Kommunen für eine einheitliche Trägerschaft der Grundsicherung für Arbeitsuchende durch die Länder bzw. durch die Kommunen - darunter $94 \%$ der in ARGEn engagierten Kreise, $100 \%$ der Optionsstädte und -kreise sowie $82 \%$ der Fälle mit getrennter Aufgabenwahrnehmung. Sollte es im Gegensatz zu diesem Votum dennoch zu einer alleinigen Bundesträgerschaft kommen, plädieren die Befragten im Übrigen erneut für eine einheitliche Zuständigkeit - dann in der Verantwortung des Bundesagentur. So findet insbesondere der seitens einiger heute noch getrennt arbeitender Träger formulierte Vorschlag, die Erwerbsfähigkeitsregelung so zu verändern, dass das SGB XII aufwachse und damit eine Kompensation zugunsten der kommunalen Zuständigkeit herstelle, keine Mehrheit: Dies lehnen 80\% der ARGEKreise, 90\% der Optionskommunen und immerhin $71 \%$ der Kreise mit getrennter Aufgabenwahrnehmung ab (Quelle: ebd.). 


\section{Ausgestaltung einer weiterhin geteilten Trägerschaft}

Für dieses Szenario präferieren $85 \%$ der befragten Kommunen $(81 \%$ der ARGE-Kreise, $100 \%$ der Optionskommunen und $67 \%$ der Kreise mit getrennter Trägerschaft) das dauerhafte Nebeneinander unterschiedlicher Trägermodelle (Quelle: ISE-Erhebung 2008). Dies sollte nach Überzeugung nahezu aller Befragten die Option einschließen, wobei $87 \%$ deren vollständige, also eine unbefristete, jederzeit mögliche und unkontingentierte Öffnung fordern. Für diejenigen Kommunen, die die eigene Vollzugsverantwortung nicht anstreben, spricht man sich unterdessen für eine (erneuerte) Kooperation mit der Bundesagentur aus, die auf verbindlichen Regelungen und Verfahren beruhen muss und möglichst die bestehenden Probleme im Personalbereich durch eine eigene Dienstherrenschaft überwinden soll. Dagegen hält man eine getrennte Aufgabenwahrnehmung mit freiwilligen Kooperationsvereinbarungen wie auch feste Koordinationsverfahren und Kooperationsangebote für nicht ausreichend, um beides einen sachgemäßen und die kommunalen Interessen wahrenden Vollzug - sicherzustellen.

Würde sich der Gesetzgeber hingegen bei geteilter Trägerschaft für ein einheitliches Trägermodell entscheiden, schlösse dies die optionale kommunale Trägerschaft aus. Erneut verbliebe dann nur die Möglichkeit eines getrennten Vollzugs mit mehr oder minder verbindlichen Koordinationsverfahren und Kooperationsangeboten seitens der BA im Bereich aktiver Hilfen - oder aber eine erneuerte ARGE bzw. obligatorische Zusammenarbeit, was wiederum eine Verfassungsänderung erforderlich machen würde. In der Tendenz dürfte eine Mehrheit der Kommunen das letztgenannte Modell bevorzugen, da man aus der Praxis heraus die materiellen und funktionalen Erfordernisse einer einheitlichen Kundenbetreuung insgesamt höher bewertet. Interessant ist in diesem Zusammenhang die Einschätzung von Varianten, die bei geteilter Trägerschaft eine operative Zusammenführung der Vollzugskompetenzen über eine Bundesauftragsverwaltung vorsehen. Das ISE hat diese Alternative bereits zu einem sehr frühen Zeitpunkt als zwar nicht vorzugswürdige, aber in eine Erörterung einzubeziehende Lösung formuliert, sofern eine einheitliche Trägerschaft oder die Fortschreibung und Öffnung der Option nicht gelingen sollte. Unter entsprechenden Umständen fände ein solches Konzept bei immerhin 55 \% der befragten Kommunen Zustimmung, setzte allerdings voraus, dass der Bund seine gesamten Kompetenzen überträgt und nicht nur (wie zuletzt von der bayerischen Staatsregierung vorgeschla- 
gen $^{5}$ ) seine Zuständigkeiten im Bereich der passiven Hilfen verlagert. Auf eine erneut klare Ablehnung stößt hingegen die Abkoppelung der Wohn- und Unterkunftskosten vom ALG II, um auf diese Weise die Nachrangigkeit des kommunalen Anteils und die operativen Verbindungen zwischen beiden Transferleistungen zu beseitigen.

\section{Zusammenfassendes Votum der Kommunen}

Die Kreise und Optionsstädte halten eine grundlegende Organisationsreform des $S G B$ II für erforderlich und votieren dabei für den Übergang zu einer einheitlichen Trägerschaft von Ländern bzw. Kommunen. Sollte es stattdessen bei geteilten Zuständigkeiten bleiben, plädieren sie für eine Fortsetzung des Systemwettbewerbs, was die Fortschreibung und vollständige Öffnung der Option sowie die Erneuerung einer kooperativen Organisationsform beinhaltet. Käme es entgegen dem kommunalen Votum zu einem (bei geteilter Trägerschaft) einheitlichen Organisationsmodell, würde man in der Tendenz eine verbundene Aufgabenwahrnehmung bevorzugen, die erforderlichenfalls über eine Verfassungsänderung zu ermöglichen wäre. Andere Lösungen werden skeptisch bis ablehnend betrachtet, so etwa die Überlegung, die Gewichte zwischen SGB II und SGB XII über eine veränderte Erwerbsfähigkeitsregelung zu verschieben, oder aber die Möglichkeit, Schnittstellen mit einer Abkoppelung der KdU vom ALG II zu beseitigen. Dies gilt auch für eine Bundesauftragsverwaltung im Bereich der passiven Hilfen nach dem bayerischen Modell. Lediglich eine vollständige Beauftragung auch mit dem aktiven Leistungsanteil des Bundes (Vermittlung und Integration) fände bei einer Mehrheit der Kommunen die erforderliche Zustimmung.

\section{Empfehlungen: Pragmatische Lösungen für ein wettbewerbsfähiges Leistungssystem}

Die nachfolgenden institutionellen und leistungspolitischen Empfehlungen beziehen die Ergebnisse der fast vierjährigen ISE-Evaluation sowie die aktuelle politische Diskussion mit ein. Dies führt die Autoren zunächst zu der Schlussfolgerung, dass für einen weiterhin erfolgreichen und reibungslosen Vollzug des SGB

5 Bayerisches Staatsministerium für Arbeit und Sozialordnung, Familie und Frauen: Neuorganisation des SGB II. Eckpunkte einer Kooperation von BA und Kommunen bei Übertragung der Geldleistungen auf die Kommunen (Stand: 05. 12. 2008), München, 2008. 
II zügig verlässliche Rahmenbedingungen geschaffen werden müssen. Aus funktionalen und systematischen Erwägungen, aber auch mit Blick auf materielle Vorteilskonstellationen und nicht belegbare Defizite, wird dabei das Modell einer flächendeckenden kommunalen Zuständigkeit grundsätzlich präferiert. Allerdings sprechen aktuelle politische Entscheidungsprobleme auf der Bundesebene und insbesondere die Schwierigkeit, eine verfassungsgemäße Lösung über eine Gesetzesausführung durch die Länder (bzw. durch die Kommunen) finanziell abzusichern, für einen pragmatischeren Handlungsansatz, der zudem die Vorteile des bestehenden Systemwettbewerbs fortschreibt. Da dies eine Öffnung der Option sowie verstärkte dezentrale Gestaltungsspielräume für die ARGEn und örtlichen Agenturgeschäftsführungen einschließen sollte, sind Verfahren vorzusehen, die dem Bund eine hinreichende Mittelkontrolle und fachliche Rahmensteuerung für seinen Leistungsanteil gestatten. $\mathrm{Zu}$ den vorgenannten organisatorischen Veränderungen sollten leistungsrechtliche Anpassungen treten, die auch unter den Bedingungen einer verschlechterten Wirtschafts- und Arbeitsmarktlage den hohen Anteil von schwer Vermittelbaren berücksichtigen und deshalb präventive wie sozialintegrative Handlungsansätze stärken. Damit verbindet sich das Erfordernis, die bisherigen Controllingsysteme und Verfahren der Erfolgsmessung im SGB II weiter zu entwickeln, um neben der bloßen Vermittlung und Kosteneffizienz auch die Nachhaltigkeit der Maßnahmen und die unterhalb einer Beschäftigung auf dem Ersten Arbeitsmarkt erfolgenden Integrationsfortschritte adäquat abzubilden. Schließlich sollen aus aktuellem Anlass jene Einschätzungen und Handlungsoptionen angesprochen werden, die im Rahmen der Fallstudien von örtlichen Akteuren mit Blick auf die gegenwärtige Wirtschaftsund Finanzkrise geltend gemacht werden, bevor eine summarische Erörterung des Zusammenhangs zwischen SGB II und gesamtstaatlicher Modernisierungsagenda verdeutlicht, dass die verfassungsgemäße Ausgestaltung der Grundsicherung für Arbeitsuchende gleichsam das „Werkstück“ einer (fortgesetzten) Föderalismusreform (auch) in der kommenden Legislaturperiode, also ab Herbst 2009, bilden könnte.

\section{Schlussfolgerungen der ISE-Evaluation zur Trägerfrage}

Die dokumentierten Ergebnisse der ISE-Evaluation der Aufgabenträgerschaft nach dem SGB II lassen keine grundsätzlichen und signifikanten Nachteilskonstellationen zulasten der Option erkennen. Dies gilt im Übrigen auch, wenn man die Kriterien der gesetzlichen $\$ 6 c$-Evaluation zugrunde legt. Soweit deren Ergebnisse bislang kommuniziert wurden, deuten sie auf keine elementaren und 
durchgehenden Defizite einer bestimmten Trägerform hin. Vielmehr ergibt sich ein gewisser Vorteil der ARGEn vor allem deshalb, weil man die Integration in eine möglichst bedarfsdeckende Erwerbstätigkeit und die hierauf bezogene Effizienz des Ressourceneinsatzes als letztlich zentrale Indikatoren ansieht. Einschränkend ist aber zu diesem Befund erneut anzumerken, dass seine Datengrundlage lediglich einen Jahreszeitraum (2006/2007) umfasst und sich zahlreiche Optionskommunen finden, die ähnlich effektiv oder sogar deutlich erfolgreicher als der Durchschnitt aller Grundsicherungsträger arbeiten, so dass ein systematisches Defizit nur schwer zu begründen ist. Hinzukommen die offenbar auch in der offiziellen Wirkungsforschung erkennbaren - und durch die ISEEvaluation bestätigten - Vorsprünge der zugelassenen kommunalen Träger im Bereich sozialintegrativer und präventiver Handlungsansätze. Somit lässt sich die Differenz zwischen den gegebenen Trägerformen derzeit wohl am besten dadurch charakterisieren, dass die ARGEn aus einer eher betriebswirtschaftlichen Logik heraus Stärken aufweisen, die Optionsstädte und-kreise dagegen vor allem im Hinblick auf ein volkswirtschaftlich und sozialpolitisch nachhaltiges Vorgehen besser abschneiden. Eine Beurteilungsbasis für eine abschließende und dauerhafte Trägerentscheidung bietet dieses Ergebnis jedoch kaum und bleibt im Wesentlichen davon abhängig, welche Kriterien man bei der Bewertung der bisher erbrachten Leistungen der Grundsicherung anlegt.

Umso mehr erscheint eine Diskussion irreführend, die bereits in einer wenig ausgewogenen und in Teilbereichen eingeschränkten Bilanz der zugelassenen Träger eine hinreichende Begründung zu erblicken glaubt, die Option gänzlich abzulehnen. So existiert weder eine gesetzliche noch eine empirische Basis, aus der sich ein solches obligo der Kreise und Städte herleiten ließe. Demgegenüber bestehen die vom ISE erkannten und von zahlreichen Beobachtern bestätigten systematischen Vorteile der kommunalen Trägerschaft und ein erhöhtes Gewicht funktionaler wie letztlich auch staatspolitischer Erwägungen. Dies berührt zunächst die durch das Verfassungsgericht festgestellte Unzulässigkeit der Mischverwaltung, wobei die Richter in ihrer Begründung erfreulicher Weise nicht nur normative, sondern auch institutionelle und funktionsorientierte Betrachtungen berücksichtigten. Hinzutreten die selbst vom Bund und seinen Evaluatoren anerkannten Vorteile einer Wettbewerbssituation zwischen ARGEn, Optionskommunen und Fällen mit getrennter Aufgabenwahrnehmung, die eine innovationsfördernde Anreizkonstellation für alle Grundsicherungsträger darstellt und entsprechende Dynamiken auslöste. Schließlich bieten im Kontext rechtlicher Vorgaben 
der bundesstaatlichen Ordnung die Besonderheiten des SGB II einen interessanten Ansatz, um an die Zielvorstellungen der Föderalismusreform II anzuknüpfen.

Im Ergebnis spricht deshalb wenig für eine voreilige und einseitige Trägerentscheidung und viel für ein besonnenes Vorgehen, das systematische Aspekte des Trägervergleichs herausarbeitet, sorgfältig gewichtet und letztlich zu einem Beschluss des Gesetzgebers führt, der den Anforderungen von Klientel und Leistungssystem gerecht wird. Hierzu gilt es, den bestehenden föderalstaatlichen Rahmen zu nutzen, mithin darauf zu verzichten, ihn entgegen der Föderalismusreform I und dem bestehenden Verwaltungsgefüge eigens für das SGB II anzupassen. Dies muss (wie bereits angemahnt) immer auch eine verlässliche Perspektive für die örtlichen Einrichtungen einschließen, also bereits während der Entscheidungsphase Interims- und Übergangslösungen beinhalten, um eine kontinuierliche Arbeitsfähigkeit zu gewährleisten.

\section{Verlässliche Rahmenbedingungen}

Dabei gilt unverändert, dass es für die weitere Entwicklung des 2005 eingeführten Leistungssystems im Interesse der Planbarkeit seitens der Träger und der Sicherheit ihrer Mitarbeiter notwendig ist, zu einem möglichst frühen Zeitpunkt abschließende Organisations- und Trägerentscheidungen zu treffen. Anderenfalls droht aufgrund von Stellenbefristungen und den damit verbundenen Unsicherheiten eine Verstetigung der bereits dargestellten Probleme. Dies spricht grundsätzlich für eine Festlegung möglichst vor 2010. Andererseits ist mit Blick auf die Bundestagswahl 2009 sowie im Interesse einer seriösen Bewertung der (bislang) vorliegenden Evaluationsergebnisse und der rechtlichen Implikationen des Karlsruher Urteils eine zügige Entscheidung ggf. nicht realistisch. In jedem Fall setzt sie auf allen Seiten die Bereitschaft voraus, an einer konstruktiven Lösung (so im Rahmen der in jedem Fall erforderlichen Personalüberführungen) mitzuwirken. Erneut würde das vor allem für die Vielzahl jener Beschäftigten einen Vorteil darstellen, deren Arbeitsverhältnis bislang auf das Ende der Experimentierphase befristet ist. Ihnen eine entsprechende Sicherheit zu bieten, würde verhindern, dass es in den kommenden Monaten zu einer verstärkten Abwanderung qualifizierten Personals kommt.

In Abwägung einer fundierten Entscheidung einerseits und der zügigen Klarstellung der organisatorischen Rahmenbedingungen andererseits plädiert der Gutachter für ein zweigleisiges Vorgehen: Zunächst sollte vermieden werden, dass es auf der Grundlage einer äußerst schmalen Datenbasis, wie im Fall der § 6c- 
Evaluation, zu einer vorschnellen und/oder einseitigen Trägerentscheidung kommt. Dies gilt umso mehr, als sich bereits anhand der bis dato bekannt gewordenen Ergebnisse eine eindeutige Modellpräferenz kaum begründen lässt. Andererseits muss für die Optionskommunen und ARGEn sowie für die Jobcenter in Fällen mit getrennter Aufgabenwahrnehmung garantiert sein, dass vorhandenes Personal von den künftig zuständigen Einrichtungen zumindest ein Übernahmeangebot erhält, soweit es nicht ohnehin ein Rückkehrrecht zu ihrem Dienstherrn besitzt. Darüber hinaus bedarf es einer Regelung für die große Zahl befristeter Beschäftigungsverhältnisse. Hier verbieten arbeitsrechtliche Sachzwänge häufig eine Verlängerung der entsprechenden Verträge, weshalb das von verschiedenen Trägern bereits praktizierte Verfahren fortzusetzen wäre, immerhin einem Teil der betroffenen Personen dauerhafte Stellen anzubieten. Berücksichtigt man, dass in den kommenden beiden Jahren aufgrund der Wirtschafts- und Finanzkrise nicht mit weiter sinkenden, sondern mittelfristig deutlich ansteigenden Fallzahlen zu rechnen ist, sollte dies unter betriebs- wie personalwirtschaftlichen Gesichtspunkten vertretbar sein.

Die damit angesprochene Interims- bzw. Übergangslösung wäre als gemeinsame Vereinbarung von Bund und kommunalen Spitzenverbänden vorstellbar. Sie sollte für eine abschließende Trägerentscheidung anschlussfähig sein bzw. in diese übergeleitet werden, sofern etwa einzelne Mitarbeiter den Dienstherrn (noch) nicht wechseln möchten und/oder die betreffenden Träger für sie keine unmittelbare Verwendung haben. In solchen Fällen wären die geltenden Abordnungs- und Gestellungsverfahren zu verlängern, bis sich die Situation über entsprechende Vereinbarungen zwischen Bund und Kommunen sowie mit den einzelnen Mitarbeitern konsolidiert hat.

Ähnliches gilt für die Vertragsdauer der ARGEn. Diesbezüglich sollte geregelt werden, dass Agenturen und beteiligte Städte wie Kreise bei einem Auslaufen ihrer Kooperationsverträge eine automatische Verlängerung $\mathrm{zu}$ unveränderten Konditionen praktizieren, bis aufgrund von Entscheidungen des Gesetzgebers klare Rahmenbedingungen geschaffen werden.

\section{Grundsätzliches Plädoyer für eine einheitliche örtliche Trägerschaft}

Die im Rahmen dieses Berichts dokumentierten Klientel- und funktionalen Leistungserfordernisse sprechen aus Sicht des ISE unverändert für eine zusammenhängende und deshalb auch möglichst einheitliche Trägerschaft unter Berücksichtigung der maßgeblichen leistungs- und integrationsspezifischen Schnitt- 
stellen zur sozialen Daseinsvorsorge (insbesondere in den Bereichen Jugend und Bildung). Dies führt trotz der unbestreitbaren Kompetenzen und Kapazitäten der Bundesagentur für Arbeit zu einem grundsätzlichen Votum für die flächendeckende Umsetzung der kommunalen Trägerschaft, da der Schwerpunkt der Betreuung von Langzeitarbeitslosen nicht allein in ihrer Vermittlung, sondern ebenso sehr in ihrer sozialen Stabilisierung zu sehen ist. Dies gilt umso mehr, als die Erfahrungen der zurückliegenden Jahre zeigen, dass es die Grundsicherungsträger auf absehbare Zeit (auch bei veränderter Wirtschaftslage) mit einem überproportional hohen Anteil schwer Vermittelbarer zu tun haben, deren Beschäftigungsfähigkeit von ihrer individuellen Stabilisierung und der ihres Umfeldes abhängt. Im Interesse einer nicht nur kurzfristigen Arbeitsmarktwirkung, sondern vor allem mit Blick auf gesamtgesellschaftliche Integrationseffekte und volkswirtschaftliche Kostenerwägungen steht daher die individuelle Betreuung und die Hebung der Arbeitsmarktnähe möglichst vieler Leistungsbezieher im Vordergrund. Genau diese strategische Ausrichtung aber ist auf den engen Schulterschluss mit anderen sozialen Dienst- wie Transferleistungen angewiesen, die von den Kommunen verantwortet werden und für deren Vollzug sie die erforderliche Problem- und Klientelnähe mitbringen.

Hinsichtlich der verfassungsrechtlichen Hürden einer Kompetenzzuweisung an die Kommunen durch den Bundesgesetzgeber müsste hierbei erforderlichenfalls der Weg einer Ausführung des SGB II als eigene Angelegenheit bzw. Auftragsverwaltung der Länder beschritten werden, was mit einer aufkommensbezogenen Anpassung der Finanzbeziehungen zwischen Bund und Ländern sowie Ländern und Kommunen zu verbinden wäre; insbesondere sollte sichergestellt werden, dass eine quotale Lösung Dynamisierungsprozesse vorsieht, die in kurzen Abständen wiederkehrende Verhandlungen über pauschale Ausgleichsbeträge weitgehend obsolet machen. Schließlich wären die Länder anzuhalten, sich im Fall einer solcher Lösung nicht - wie heute die Bundesagentur - detailliert in den materiellen Vollzug einzuschalten, da ansonsten Vorteile, die eine eigenständige Aufgabenwahrnehmung vor Ort bieten, in einer den ARGEn vergleichbaren Form konterkariert würden.

\section{Pragmatische Lösung über eine Öffnung der Option}

Trotz der aus normativen wie systematischen Gründen heraus als vorzugswürdig vorgestellten Lösung sprechen staatspolitische, fiskalische wie funktionale Erwägungen doch für einen Mittelweg bzw. ein kompromissorientiertes Vorgehen. So ist zunächst auch als Ergebnis der ISE-Evaluation nicht zu bestreiten, dass 
zahlreiche ARGEn erfolgreich und professionell arbeiten, insbesondere dann, wenn ihnen die erforderlichen dezentralen Freiheiten gewährt werden und sich personelle Konstellationen herausgebildet haben, die institutionelle Hemmnisse überbrücken. Hinzutritt in strukturschwachen Regionen und Großstädten die zwar nicht zwangsläufige, aber doch nachvollziehbare Erwägung, nach der der Problemumfang bei zugleich schwierigen Arbeitsmarktbedingungen die Kommune überfordern könnte, wenn sich der Bund nicht mehr in einer aktiv gestaltenden Mitverantwortung sieht. Zudem erscheint es politisch außerordentlich zweifelhaft, dass die Länder ihre seit dem Karlsruher Urteil auf der A- wie der B-Seite erkennbare Zurückhaltung gegenüber einer fiskalischen Umschichtung aufgeben und auch der Bund dem zu seinen Lasten zustimmt. Schließlich sind die positiven Effekte der Modellkonkurrenz zu beachten, die sich durch den Wettbewerb reiner Agentur-, kooperativer und allein kommunaler Lösungen ergeben haben. Sowohl auf Agentur- als auch auf Kreis- wie städtischer Seite führte der permanente Vergleich zu einer erhöhten Motivation und Innovationsdynamik, die im deutschen Kontext so am ehesten im kommunalen Bereich und i. T. zwischen den Ländern anzutreffen ist. Mit anderen Worten: Gerade die Experimentierphase mit unterschiedlichen Organisationsmodellen dürfte entscheidend zu der positiven Bilanz des neuen Leistungssystem beigetragen haben. Somit erscheint es auch aus funktionalen Gründen gerechtfertigt, die gegebenen Systemvorteile mit den benannten Vorzügen einer dezentralen Zuständigkeit zu verbinden.

Der Gutachter plädiert deshalb für ein Konzept, das möglicherweise ohne Verfassungsänderung auskommt, die örtlichen Trägerstrukturen absichert (bzw. funktional optimiert) und die angesprochenen Wettbewerbsvorteile gewährleistet. Die Basis hierfür bildet die vollständige Öffnung der Option, während die Bildung einer $A R G E$ im SGB II für die kommunale Seite als Kann-Regelung ausgestaltet wird. Dies hätte zur Folge, dass weitere Kommunen die zugelassene Trägerschaft wählen könnten. Um auch der weiterhin verpflichteten Agentur bzw. Bundesseite in dieser Konstellation hinreichende Planungssicherheit zu gewähren, sollte der Modellwechsel mit terminlichen und personalwirtschaftlichen Konditionen verbunden werden. So wäre die Option nach dem bislang geltenden Zulassungsund Genehmigungsverfahren zu ,ziehen“ und wenigstens für einen bestimmten Zeitraum von etwa vier bis fünf Jahren auszuüben. Ähnliches gälte für die Bildung einer ARGE (in Form einer abzuschließenden Vereinbarung) oder die getrennte Aufgabenwahrnehmung. Demnach stünden die Kommunen in der Pflicht, für eine definierte Konzessionsphase zu entscheiden, ob sie den Vollzug des 
SGB II in eigener Verantwortung übernehmen, gemeinsam mit der Agentur erledigen oder aber hiervon nur ihre gesetzlichen Trägerfunktionen erfüllen wollen. ${ }^{6}$ Dabei gilt unverändert, dass Modellwechsel die bereits angesprochenen Personalverpflichtungen nach sich ziehen müssten, also die übernehmenden Einrichtungen den bislang im Bereich Beschäftigten eine Tätigkeit anzubieten hätten und dies (fortgesetzte) Abordnungen oder Personalgestellungen einschließen könnte.

Der angestrebte Wettbewerbseffekt, den ein solches System auslösen würde, sollte sich in dreifacher Hinsicht einstellen: Zunächst dürfte der permanente Vergleich zwischen den unterschiedlichen Modellen die geschilderten positiven Anreizwirkungen verstetigen, zudem fände sich die kommunale Stellung in den ARGEn oder in getrennten Konstellationen gestärkt, da zumindest perspektivisch die eigene Trägerschaft als Alternative zur Verfügung stünde, und käme es schließlich zu einem Dezentralisierungsschub innerhalb der Agenturen, da diese fortan nur dann adäquat auf ihre kommunalen Verhandlungspartner reagieren können, wenn sie über eigenverantwortlich wahrzunehmende Gestaltungsspielräume verfügen.

Hinzutreten sollte im Interesse einer Stabilisierung der ARGEn die Möglichkeit einer eigenen Dienstherrenschaft der gemeinsamen Einrichtungen. Dies würde endlich jene Planungssicherheit und Entwicklungsfähigkeit ermöglichen, der die getrennten Personalkörper bislang entgegenstehen. Die zugrunde liegenden Vereinbarungen zur Gründung dieser neuen ARGEn müssten Regelungen für den Fall beinhalten, dass die Einrichtungen (nach einem Modellwechsel) zu einem späteren Zeitpunkt nicht als kooperative Einheit fortgeführt werden sollen. Innerhalb der Bundesagentur wäre ferner zu erwägen, die Zuständigkeit für das SGB II als eigenen durchgängiger Organisationsstrang zu etablieren. Dies würde zumindest in Fällen mit ARGEn oder bei getrenntem Vollzug örtliche SGB II-

6 Ergänzend wäre (gleichsam als Kompromisslinie) denkbar, die Zahl der Optionskommunen auf maximal (oder weniger als) die Hälfte der Städte und Kreise zu begrenzen, um einerseits den Charakter der Option als Ausnahmetatbestand aufrecht zu erhalten und andererseits dem Bund und der BA eine volumenmäßige Planungssicherheit für die im SGB II einzusetzenden Verwaltungs- und Personalmittel zu geben. Sollte dieses Kontingent erschöpft sein, wären neuerliche Optionen erst dann möglich, wenn bestehende zurückgegeben würden oder infolge von Gebietsreformen wegfielen. Wie ausgeführt, hält der Gutachter diese Lösung nur für eine second best-Variante, die aber der politischen Kompromissfindung dienen könnte, zumal die bereits weiter oben empfohlene Übergangs- bzw. Interimsvereinbarung dauerhaft vermeiden sollte, dass Trägerentscheidungen einseitige finanzielle Lasten beim Bund oder den Kommunen auslösen. 
Geschäftsführer und auf regionaler Ebene entsprechende Direktionsvertreter erfordern.

\section{Erhöhung der dezentralen Gestaltungsmöglichkeiten}

Unabhängig von der Trägerfrage und einer inhaltlichen Erweiterung des SGB II sollte es den örtlichen Einrichtungen in erheblichem Maße gestattet sein, eigene Eingliederungsinstrumente zu entwickeln und auf die jeweiligen Klientelbedürfnisse anzupassen. Bis zu der Festlegung restriktiverer Vorgaben für die sonstigen weiteren Leistungen in den Jahren 2007 und 2008 durch das BMAS und die BA bot hierfür vor allem der $\S 16$ Abs. 2 Satz 1 grundsätzlich ausreichende Möglichkeiten. Das Gesetz zur Neuausrichtung der arbeitsmarktpolitischen Instrumente könnte die entstandene Lücke schließen, sofern nicht der Vollzug erneut sehr engen Regeln unterworfen wird - was als Aufforderung an den Bund zu richten wäre. Darüber hinaus würde es sich in Ergänzung zu den $\S \S 16 \mathrm{~d}$ (früher $\S 16$ Abs. 3) und 16e (früher § 16a) anbieten, die Nutzung von Kapitalisierungsvarianten für passive Hilfen im Rahmen von öffentlicher Beschäftigung, Kombilohnsystemen und Subventionsformen zu ermöglichen und ggf. die Grenzen zwischen Eingliederungs-, Verwaltungs- und Leistungsbudgets generell zu lockern bzw. aufzuheben.

Hinzutreten müssten schließlich längerfristig verlässliche Budgetzusagen des Bundes und die Übertragbarkeit der im abgelaufenen Jahr ungenutzten Mittel. Speziell für die Situation der ARGEn wäre zu empfehlen, den örtlichen Agenturgeschäftsführungen mehr operative Zuständigkeiten und Spielräume bei der Wahrnehmung der Trägerfunktion einzuräumen, da auf diese Weise Spannungen im Verhältnis zur Kommune abgebaut und die dezentrale Beweglichkeit im Vollzug erhöht werden könnten - dies korrespondiert mit der beschriebenen Verstetigung des Systemwettbewerbs infolge einer Öffnung der Option. Darüber hinaus sollten Bundesagentur und Kommunen die schwierigen Personalkonstellationen in den ARGEn mittels einer angenäherten Besoldungs- und Tarifstruktur zu überwinden suchen. Dies gilt erneut auch für die bislang beschränkte Personalentwicklung und die infolgedessen reduzierte Attraktivität einer Arbeit in den Jobcentern. Insbesondere dann, wenn man sich im Zuge einer Reform der Trägerstrukturen nicht auf die oben vorgeschlagene Möglichkeit einer eigenen Dienstherrenfähigkeit der ARGEn einigen sollte, wäre es zumindest erforderlich, seitens der BA und der beteiligten Kommunen den Geschäftsführungen Personalentwicklungskontingente in Form von Hebungs- und Beförderungsmöglichkeiten einzuräumen. 


\section{Steuerungsinteressen des Bundes}

Die vom ISE vorgetragenen Empfehlungen zielen auf eine Dezentralisierung und Flexibilisierung der Vollzugsverantwortung im SGB II. Insbesondere dann, wenn es nicht zu der systematisch vorzugswürdigen Lösung einer Verwaltung als Ländersache kommt, sondern der pragmatische Weg eines verstetigten Systemwettbewerbs beschritten wird, bliebe es dem Grunde nach bei der gegenwärtigen Aufgaben- und Finanzierungsträgerschaft. Das heißt, dass weiterhin der Bund für einen übergroßen Ausgabenanteil aufzukommen hat, der wiederum eine wesentliche Quote des gesamten Staatshaushaltes ausmacht. Es muss von daher als Selbstverständlichkeit gelten, dass hierüber eine angemessene Kontrolle auszuüben ist. Dies gebietet bereits das Haushaltsrecht und lässt sich auch verfassungssystematisch begründen. Hinzutreten politische Erfordernisse, da eine gebietskörperschaftliche Ebene und ihre Entscheider nicht darauf verzichten können, die mit entsprechenden Finanzmitteln verbundenen Einflusspotenziale zu nutzen, zumal sie (über weniger populäre Einnahme- und Verteilungsentscheidungen) die dafür notwendigen Ressourcen mobilisieren müssen. Insofern erweisen sich die vom Bund (und in seinem Namen vom BMAS und von der Bundesagentur für Arbeit) geltend gemachten Steuerungsansprüche nicht nur als nicht abwegig, sondern im Kern als berechtigt und kaum bestreitbar.

Gleichwohl bestehen mit Blick auf die bisherige Verwaltungspraxis im neuen Leistungssystem offenbar sehr unterschiedliche Einschätzungen, welche Form und Intensität zentralstaatlicher Steuerung der Gesetzgeber für das SGB II vorsieht bzw. vorsehen sollte. Zwar ist die Differenzierung zwischen strategischer und operativer Verantwortung allenthalben anerkannt und fehlt sie in keiner Darstellung auch des Bundes, doch erscheinen seine Vorstellungen und die der kommunalen Partner zur operativen Umsetzung dieser Steuerungsphilosophie sehr uneinheitlich, in weiten Teilen sogar gegensätzlich. Auch stellt sich die Frage, inwieweit bestehende Routinen und Instrumente überhaupt dazu geeignet sind, strategische Steuerung und ein Mindestmaß an (bundes-)haushaltsrelevanter Kontrolle auszuüben, und ob hierzu neben den regulären Gesetzes-, Verordnungs- und Aufsichtsmitteln ausdifferenzierte Weisungssysteme und Prüfverfahren erforderlich und zulässig sind. Schließlich muss vor diesem Hintergrund befunden werden, welche Veränderungen notwendig sind, um die materiell gebotene Dezentralisierung (i.S. einer klientel- und problemnahen Stabilisierung bzw. Integration von Langzeitarbeitslosen) mit der notwendigen Rahmensteuerung und Ressourcenkontrolle (als Ausfluss der Gesetzgebungskompetenz und der Finanzierungspflicht des Bundes) funktionsfähig zu verbinden. 
Zunächst gilt für die Ausgestaltung einer zentralen Rahmen- und örtlichen Umsetzungsverantwortung, dass auf beiden Seiten nennenswerte Gestaltungsspielräume bestehen müssen. Hinzutritt - zumal in dem vom Gutachter empfohlenen System - die Berücksichtigung der besonderen Handlungsmuster der beteiligten Partner. Strategische Vorgaben sind demnach auf der Zielebene anzusiedeln, mit der Möglichkeit, sie regional wie lokal unterschiedlich zu erfüllen und in diesem Rahmen eigene Schwerpunkte zu setzen. Kommunaler Selbstverwaltung ist eine solche Vorgehensweise auch unter den Bedingungen des übertragenen Wirkungskreises inhärent, wobei die Weisung seitens übergeordneter Instanzen den Einzelfall bilden sollte. Entsprechende Zielsetzungen hat der Bund im SGB II klar formuliert. Mit dem vorliegenden Bericht wird nicht zuletzt deshalb angeregt, diese Orientierung um eine verstärkte Rolle von sozialer Integration und Prävention zu ergänzen. Einer solchen Empfehlung zu entsprechen oder aber am bestehenden Vermittlungsprimat festzuhalten, wäre demnach ein Beispiel für die Ausübung der strategischen Steuerungskompetenz des Bundes. Sie schließt darüber hinaus aber auch die Prioritätensetzungen zugunsten bestimmter Zielgruppen ein (U25 und Ü55 als Beispiele), die wiederum über Mittelentscheidungen und das Instrumentendesign weiter konkretisiert werden. Insbesondere Letzteres stellt eine wesentliche, bereits heute sehr weit ausdifferenzierte Möglichkeit zur Ausrichtung der Grundsicherung im Bereich aktiver Hilfen dar, was mit dem Gesetz zur Reform arbeitsmarktpolitischer Instrumente bestätigt wurde. Insofern verfügt der Bund als Gesetzgeber bereits über entscheidende Stellschrauben, mit denen er das Leistungssystem steuern kann.

Darüber hinaus beansprucht er allerdings gerade in den ARGEn weitergehende Weisungskompetenzen, die in seinem Auftrag die Bundesagentur exekutiert. Blickt man etwa auf die zuletzt veröffentlichten Vorgaben zu den Stellenschlüsseln, dürfen diese als außerordentlich weitgehend und in einem personalgebundenen Dienstleistungsbetrieb auch als essentiell für die Ausgestaltung dezentraler Organisationsstrukturen und Abläufe bezeichnet werden. Hier von einer unverändert bestehenden Handlungsautonomie vor Ort zu sprechen, erscheint im Lichte solcher Direktiven wenig nachvollziehbar. Insofern ist damit beispielhaft ein Punkt benannt, an dem zentrale und dezentrale Verantwortungsbereiche kollidieren und in einem System dezentralen Wettbewerbs notwendige Grenzen überschritten werden. Binnenorganisation und Personaleinsatz sollten danach von Mindeststandards abgesehen (etwa als Korridore für die Betreuungsrelationen für bestimmte Zielgruppen und Grundparameter) in der alleinigen Verantwortung der örtlichen Einrichtungen liegen. Dies gilt - wie ausgeführt - auch für 
die Budgetgestaltung und den Instrumenteneinsatz. Gerade wenn der letztgenannte Bereich über differenzierte Vorgaben im Gesetz hinaus weiter reglementiert wird, stellt sich nicht nur die Frage, inwieweit die örtlichen Ermessensspielräume zunehmend einer fachrechtlichen Kombinatorik und Exegese weichen, sondern ob Systeme mit Zielvereinbarungen überhaupt noch geeignete Handlungsformen darstellen oder letztlich ein weisungsgebundenes Verhältnis lediglich überformen, ggf. sogar weiter verkomplizieren. ${ }^{7}$ Mit anderen Worten: Will man dezentrale Vollzugsautonomie im Rahmen strategischer Ziele erhalten, muss man sich seitens der zentralen Steuerungsinstanz zurücknehmen. Anderenfalls erscheint ein klar und vollständig hierarchisches Modell vernünftiger, da es Schwankungen im Führungsstil und somit Unsicherheiten für den Vollzug vermeidet. Genau Letzteres ist vor allem in den ARGEn als verbreiteter Eindruck feststellbar. Den materiellen Erfordernissen des SGB II und auch den Aussagen der politisch Verantwortlichen entspricht dies nicht.

Für das hier empfohlene System eines fortgesetzten Modellwettbewerbs erscheint es daher dringend erforderlich, die Steuerungsmittel des Bundes auf herkömmliche, vor allem klar interpretierbare und sinnvoll anwendbare Handlungsformen zu konzentrieren. Hierzu steht zunächst das seit seiner Einführung wiederholt angepasste SGB II selbst zur Verfügung. Wie auch im Rahmen der vorliegenden Evaluation nachvollziehbar ist, erwies sich das Sozialgesetzbuch II als durchaus steuerungsstark, kam es doch tatsächlich zu einer bevorzugten Betreuung von U25-Kunden, wurde flächendeckend ein Fallmanagement eingeführt und dem Primärziel einer Vermittlung in den Ersten Arbeitsmarkt (bei aller Kritikwürdigkeit der einseitigen Fixierung auf dieses Ziel) erkennbar höchste Priorität eingeräumt. Umso mehr erscheint es gerade im Vergleich von ARGEn und Optionskommunen wenig überzeugend, wenn jenseits personeller Kapazitäten und routinebehafteter Strukturen die Übersteuerung durch die Bundesagentur als wesentlicher Beitrag zum Handlungserfolg gemeinsamer Einrichtungen angesehen wird. Stattdessen wäre es zu bevorzugen, die Anzahl an Vorgaben und Geschäftsanweisungen innerhalb der BA erkennbar zurückzuführen und an transparente wie strenge Kriterien $\mathrm{zu}$ binden. Zugleich sollten unbedingt notwendige Konkretisierungen des SGB II allgemeinverbindlich für alle Träger und Grundsicherungsstellen über eine jährliche Ausführungsverordnung des BMAS realisiert werden, erforderlichenfalls mit (Teil-)Zustimmungspflicht des Bundes-

7 Zu der häufig sehr ambivalenten Funktion und Wirkung von Zielvereinbarungen vgl. auch die Ausführungen bei Hesse, J.J./Götz, A.: Gesetz zur Weiterentwicklung der Verwaltungsstrukturreform, a. a. O. 
rats. Zwar ist der diesbezügliche Handlungsbedarf grundsätzlich als begrenzt einzuschätzen, doch dürfte es sich bereits systematisch und funktional als Vorteil erweisen, wenn sich entsprechende Vorgaben formal und zeitlich in einem Regelungsinstrument zusammenfassen ließen. Im Übrigen könnte dies dann auch das Verhältnis zwischen BMAS und den (nach den hier unterbreiteten Vorschlägen künftig zahlreicheren) Optionskommunen rationalisieren und Auseinandersetzungen über nachholende Vollzugs- und Finanzkontrollen vermindern, diese ggf. sogar im bisherigen Umfang überflüssig machen.

Gleichwohl bildet der letztgenannte Aspekt der Mittel- und Verwendungskontrolle ein weiteres berechtigtes Bundesinteresse, das alle Beteiligten - auch die zugelassenen Träger - zu berücksichtigen haben. In diesem Kontext sind die heute seitens der Prüfgruppe des BMAS bestehenden Kompetenzen im Kern nicht zu beanstanden. Gelegentliche Friktionen und Konflikte dürften sich in den betreffenden Verfahren im Übrigen vor allem dann reduzieren lassen, wenn der Gesetzgeber eine klarstellende Entscheidung zur künftigen Trägerstruktur getroffen hat. Ungeachtet dessen bleibt es dabei, dass die gegebenen Berichts- und Prüfansprüche (und -pflichten) des Bundes legitim und notwendig sind. Dies gilt im Übrigen auch für gemeinsame Standards, Statistikpflichten und Controllingverfahren, worauf im Folgenden noch einmal gesondert eingegangen wird.

\section{Präventive und sozialintegrative Konkretisierung des SGB II}

Unverändert erforderlich erscheint die leistungsseitige Absicherung der empfohlenen Reformen über die Einführung und vor allem Konkretisierung eines präventiven und sozialintegrativen Handlungsauftrages in das SGB II. Dies entspräche der inzwischen von allen großen Parteien geforderten Adjustierung des deutschen Sozialstaats, der künftig weniger nach- und in stärkerem Maße vorsorgend tätig werden sollte. Hierzu bedürfte es der Verpflichtung der örtlichen Träger, neben der Vermittlung und Herstellung der Beschäftigungsfähigkeit den Abbau individueller Hemmnisse und Problemlagen auch dann zu betreiben, wenn keine kurz- oder mittelfristigen Aussichten bestehen, die betreffenden Klienten in eine reguläre Beschäftigung auf dem Ersten Arbeitsmarkt zu vermitteln. Zugleich müssten im Rahmen der bestehenden (oder entsprechend erweiterten) Budgets die Voraussetzungen dafür geschaffen werden, um Mittel zugunsten solcher Personenkreise einzusetzen, die perspektivisch von Hilfebedürftigkeit betroffen sein können. Beides - die sozialintegrative Profilierung und auch präventive Ausrichtung des SGB II - dürfte sich mittel- und langfristig als wirksamere Strategie bei der Reduzierung von Leistungsempfängern und Erwerbslosen 
sowie der mit ihnen verbundenen Folgekosten erweisen als reine Vermittlungsansätze, selbst wenn hierfür zunächst höhere Transferausgaben und Eingliederungsmittel vorzusehen wären. Schließlich sind die Grundsicherungsträger aufgerufen, auch über die Grenzen von SGB III und SGB II hinweg stärker zusammenzuarbeiten. So könnten ARGEn wie Kommunen Betreuungskunden im ALG I-Bezug (ohne ergänzende Leistungsansprüche nach dem SGB II) bereits sehr viel früher übernehmen und damit von der Agentur beauftragt werden. Auf diese Weise würden eine höhere Kontinuität bei der Betreuung gewährleistet und Ruhephasen vermieden.

\section{Statistik und Erfolgsmessung}

Die angesprochene sozialintegrative und präventive Erweiterung der materiellen Zielsetzungen des SGB II muss sich in den Systemen zur Statistik und Erfolgsmessung wieder finden. Dabei sind ein gemeinsamer Rahmen und verbindliche Verfahren allein schon deshalb unverzichtbar, um die Fremdverwaltung von erheblichen Ressourcen durch die Kommunen im Fall der Option abzusichern. Im Übrigen sprechen dafür aber auch ordnungspolitische Gründe, da Informationen über den Stand der Aktivitäten der Grundsicherung, wie insbesondere über deren Wirkung zentrale Grundlagen einer Rahmensteuerung durch den Bund und der wettbewerbsnotwendigen Vergleichsfähigkeit zwischen den örtlichen Einrichtungen darstellen. Umso wichtiger erscheint vor dem Hintergrund der geltend gemachten sozialpolitischen Ergänzungsbedarfe die Installation eines Systems, das verlässlich auch längerfristig Integrationsfortschritte unterhalb der Vermittlungsschwelle in den Ersten Arbeitsmarkt abbildet und die Beständigkeit einer Beschäftigungsaufnahme misst.

Berücksichtigt man die Schwierigkeiten, die sich hierbei mit Blick auf die Objektivität individueller Angaben auf Sachbearbeiter-wie Fallmanagement-Ebene ergeben, wird dies eine dauerhafte externe Unterstützung erforderlich machen, die neben statistischen Methoden ggf. auch qualitative Ansätze einbezieht. Hinzutritt das Erfordernis, die erweiterte Zielstellung des SGB II über ein gleichermaßen arrondiertes set an äquivalenten Kennzahlen und Indikatoren zu dokumentieren, also etwa neben Vermittlungen und Kosten pro Integration auch SGB II-Quoten, differenzierte kommunale Ausgabenanteile und ehB-Zahlen sowie Veränderungen bei der Summe der Leistungsempfänger aufzunehmen und diese mit handlungsleitenden Prioritäten zu versehen. 
Für die künftige Ausgestaltung im Rahmen eines dezentralisierten Vollzugs und verstetigten Modellwettbewerbs folgt daraus, dass Statistikpflichten gesetzlich fixiert bleiben müssen, während Identität und Auswahl von Indikatoren durch gemeinsame Gremien von Bund, Ländern und Kommunen verantwortet und weiterentwickelt werden sollten. Hinzuträte notwendiger Weise eine externe und - im Interesse wechselseitiger Akzeptanz - auch unabhängige Begleitung der statistischen und qualitativen Erfolgsmessung. Dies könnte die gesamte Arbeitslosenstatistik einbeziehen, zumal hier unverändert Vorbehalte erkennbar sind, auch wenn sich Skandale, wie vor dem Umbau der Bundesagentur, bislang nicht wiederholt haben - eine Option böte etwa die Ansiedlung beim Statistischen Bundesamt. Gleichwohl erscheint dieser umfassende und die Agentur in einem ihrer Kernbereiche berührende Schritt nicht so notwendig wie die zuvor benannte externe Erfolgsmessung im Kontext einer anspruchsvolleren Bewertung des SGB II-Vollzugs und seiner Auswirkungen.

Deshalb empfiehlt der Gutachter zunächst die Bildung eines Gemeinsamen Norm- und Standardausschusses von Bund, Ländern und kommunalen Spitzenverbänden sowie die dauerhafte Beauftragung externer Sachverständiger, die auf der Basis von BA- und selbst erhobenen Daten sowie unter Nutzung von örtlichen Informationen der einzelnen Grundsicherungsstellen ein für das Controlling geeignetes Monitoring aufbauen, um Integrationserfolge i.w.S. und deren Ressourceneffizienz zu dokumentieren. Der damit verbundene Aufwand wäre zwar erheblich, doch ein vor dem Hintergrund der hohen politischen Bedeutung akzeptabler Preis, zumal auf diesem Wege bestehende Konflikte zu vermeiden wären und man zu einem letztlich objektiveren Verfahren gelangen könnte.

\section{Reaktion auf die internationale Wirtschafts- und Finanzkrise}

Nach Einschätzungen der vor Ort befragten Grundsicherungsträger, rechnen die handelnden Akteure vor Ort noch nicht mit einem wesentlichen Anstieg der Fallzahlen. Gleichwohl wird es etwa in der Gruppe der Aufstocker und bei Personen mit kurzen ALG I-Bezugsrechten möglicherweise schneller als erwartet zu einer Zunahme kommen. Insofern muss sich auch der SGB II-Bereich auf entsprechende Probleme einstellen, zumal sich die Aufnahmefähigkeit des Ersten Arbeitsmarktes im Jahresverlauf 2009 und wohl ebenso in 2010 reduzieren dürfte. Reaktionsmöglichkeiten bestehen hier in der auch bereits von der BA formulierten Priorität, Qualifizierung und Weiterbildung voranzutreiben. Hinzutritt das Erfordernis, schwer Vermittelbare nicht ,abzuhängen“, mithin an den auch in diesem Bericht formulierten Empfehlungen zu einer verstärkten sozialintegrati- 
ven Orientierung festzuhalten, da diese jene Umfeldstabilisierung ermöglichen, die mittel- und langfristig den Anteil problembehafteter Personen zu reduzieren hilft. Darüber hinaus gilt es, für neu oder erneut in das Hilfesystem gelangende Menschen ein Absinken der Arbeitsmarktnähe und der Beschäftigungsfähigkeit während des Leistungsbezugs zu vermeiden. Gerade aber für den Fall, dass die Nachfrage nach dieser Klientel vorerst geringer ausfällt, dürfte es sich als unvermeidlich erweisen, ergänzend zu den benannten Qualifizierungsmaßnahmen öffentliche Beschäftigungsansätze zu verstärken. So könnte eine Möglichkeit darin bestehen, für einen zunächst begrenzten Zeitraum den Einsatz von Arbeitsgelegenheiten auf zwei Jahre zu verlängern. Freilich wäre hierbei seitens der Grundsicherungsträger darauf zu achten, dass sich die Tätigkeit als „Ein-EuroJobber" mit einer intensiven Begleitung durch das Fallmanagement oder beauftragte Dritte verbindet. Inwieweit ferner ein dauerhaft zu installierender öffentlicher Beschäftigungssektor angezeigt ist, muss derzeit noch nicht entschieden werden, sofern man sich auf einen erweiterten, ggf. auch befristeten Einsatz der vorliegenden Instrumente verständigt; die Maßnahmen nach § 16e (früher § 16a) bieten zusätzliche Möglichkeiten.

\section{Modernisierung des SGB II als Teil der gesamtstaatlichen Reformagenda}

Eine nach den Grundsätzen der Prävention, sozialen Integration sowie Orts- und Problemnähe vorgenommene Weiterentwicklung des SGB II würde in mehrfacher Hinsicht zur gesamtstaatlichen Modernisierung beitragen, zumal nach der ersten Stufe der Föderalismusreform in einem zweiten oder auch weiteren Schritten verwaltungsorganisatorische und finanz(verfassungs)rechtliche Fragen der künftigen bundesstaatlichen Ordnung erörtert werden müssen. ${ }^{8}$ Materiell verbände sich dies zunächst mit einer größeren gesellschaftspolitischen Nachhaltigkeit, die aus einer verbesserten Familien- und Kinderförderung, der Aufhebung bzw. Verringerung herkunftsabhängiger Bildungs- und Berufschancen und der Vermeidung finanziell wie individuell problematischer Folgekosten durch vermehrte Investition in Lernfähigkeit und Qualifikation resultierte. Hinzuträte eine sozialpolitische Stabilisierung aufgrund der Umsteuerung des bislang primär passiven und nachsorgenden Hilfeansatzes hin zu einer vorsorgenden, Betroffene integrie-

8 Vgl. hierzu die Stellungnahme im Rahmen der 8. Sitzung der Kommission zur Modernisierung der Bund-Länder-Finanzbeziehungen (Föderalismusreform II - Anhörung vom 08.11. 2007): Hesse, J.J.: Stellungnahme im Rahmen der Sachverständigen-Anhörung der Kommission von Bundestag und Bundesrat zur Modernisierung der Bund-Länder-Finanzbeziehungen, K-Drs. 078, Berlin, 2008. 
renden und aktivierenden Leistungspolitik des Sozialstaates. Schließlich käme es im Gefolge der skizzierten Veränderungen zu einer staatspolitischen Modernisierung, da zumindest sektoral (also im Bereich der Arbeitsmarkt- und Sozialpolitik) eine Entflechtung der gebietskörperschaftlichen Ebenen sowie eine fachspezifische Deregulierung und Entbürokratisierung der öffentlichen Aufgabenwahrnehmung erreicht würden. Dies mündete in ein erwartbar positives Beispiel für innerföderalen wie intersektoralen Wettbewerb. Aufgrund der großen politischen und öffentlichen Wahrnehmung des SGB II, des erheblichen Finanzvolumens der damit verbundenen Leistungen sowie der Verknüpfung unterschiedlicher Reformthemen könnte die Modernisierung der Grundsicherung für Arbeitsuchende somit auch in dieser Hinsicht als ,Gelenk- und Werkstück“ einer zeitgemäßen Weiterentwicklung sozialer Leistungssysteme und der bundesstaatlichen Ordnung dienen, zumal gerade letztere im Spannungsfeld zwischen zentral- und gliedstaatlichen Interessen einmal mehr nur kleinteilig auszufallen droht. 